\title{
Article
}

\section{Stochastic dynamic for an extensible beam equation with localized nonlinear damping and linear memory}

\author{
Abdelmajid Ali Dafallah ${ }^{1, *}$, Fadlallah Mustafa Mosa ${ }^{2}$, Mohamed Y. A. Bakhet ${ }^{3}$ and Eshag Mohamed \\ Ahmed $^{4}$ \\ 1 Faculty of Petroleum and Hydrology Engineering, Alsalam University, Almugled, Sudan. \\ 2 Department of Mathematics and physics, Faculty of Education University of Kassala Kassala, Sudan. \\ 3 Department of Mathematics, College of Education Rumbek University of Science and Technology Rumbek, South \\ Sudan. \\ 4 Faculty of Pure and Applied Sciences, International University of Africa, Khartoum, Sudan. \\ * Correspondence: majid_dafallah@yahoo.com
}

Received: 28 July 2020; Accepted: 7 October 2020; Published: 30 November 2020.

\begin{abstract}
In this paper, we concerned to prove the existence of a random attractor for the stochastic dynamical system generated by the extensible beam equation with localized non-linear damping and linear memory defined on bounded domain. First we investigate the existence and uniqueness of solutions, bounded absorbing set, then the asymptotic compactness. Longtime behavior of solutions is analyzed. In particular, in the non-autonomous case, the existence of a random attractor attractors for solutions is achieved.
\end{abstract}

Keywords: Beam equation, memory, nonlinear damping, Random Dynamical System, random attractor.

MSC: Primary 35Q35, Secondary 35B40, 35B41, 35B45.

\section{Introduction}

e consider the following extensible beam equation with localized non-linear damping and linear memory on a bounded domain:

$$
\left\{\begin{array}{l}
u_{t t}+\Delta^{2} u-k(0)\left(1+\int_{\Omega}|\nabla u|^{2} d x\right) \Delta u-\int_{0}^{\infty} k^{\prime}(s) \Delta u(t-s) d s+a(x) g\left(u_{t}\right)+f(u)=q(x, t)+\kappa \sum_{j=1}^{m} h_{j} \dot{W}_{j}(t) \\
u=\frac{\partial u}{\partial \Gamma}=0, \quad x \in \partial \Gamma, t \in \mathbb{R} \\
u(\tau, x)=u_{0}(\tau, x), u_{t}(\tau, x)=u_{1}(\tau, x), x \in \Gamma, \tau \in \mathbb{R}
\end{array}\right.
$$

where $\Gamma$ is a bounded domain of $\mathbb{R}^{n}, k(0), k(\infty)>0$ and $k^{\prime}(s) \leq 0$ for every $s \in \mathbb{R}^{+}, \varepsilon$ is a positive constant. The given function $g(x, t) \in \mathrm{t}_{\text {loc }}^{2}\left(\mathbb{R}, L^{2}(\Gamma)\right)$ is a external force depending on $\mathrm{t}, h_{j} \in H^{2}(\Gamma)$ and $W(t)$ is an independent two sided real-valued Wiener processes on probability space. The function $a(x)$ satisfies

$$
a(x) \in L^{\infty}(\Gamma), a(x) \geq \alpha_{0}>0, \quad \text { in } \Gamma
$$

where $\alpha_{0}$ is constant. The function $f \in C^{1}(\mathbb{R})$ satisfies

$$
\begin{cases}\left(\mathbf{A}_{1}\right): & \left|f^{\prime}(s)\right| \leq C_{1}\left(1+|s|^{\gamma-1}\right), \forall s \in \mathbb{R}, \\ \left(\mathbf{A}_{2}\right): & \liminf |s| \rightarrow \infty \frac{|f(s)|}{s}>-\lambda_{1}, \\ \left(\mathbf{A}_{3}\right): & F(s)=\int_{0}^{s} f(r) d r \geq C_{2}\left(|s|^{\gamma+1}-1\right), \\ \left(\mathbf{A}_{4}\right): & s f(s) \geq C_{3}(F(s)-1), \\ \left(\mathbf{A}_{5}\right): & C_{2}\left(|s|^{\gamma+1}-1\right) \leq F(s) \leq \frac{1}{C_{3}}\left(s f(s)+C_{3}\right),\end{cases}
$$

where $C_{i}$ are positive constants $(i=1,2,3,4), 1 \leq \gamma \leq \frac{n+2}{n-2}, n \geq 3$ and $\lambda_{1}$ is the best constant in the Poincáre-type inequality

$$
\lambda_{1} \int_{\Omega}|u|^{2} d x \leq \int_{\Omega}|\nabla u|^{2} d x .
$$


The damping function $g$ satisfies $\left|g^{\prime}(s)\right| \geq 0, g(s)$ strictly increasing, and

$$
|h(0)|=0,0<\alpha_{1} \leq\left|h^{\prime}(s)\right| \leq \alpha_{2}<\infty .
$$

As like to $[1,2]$, we define a new variable

$$
\eta(x, t, s)=u(x, t)-u(x, t-s), \eta_{t}=\frac{\partial}{\partial t} \eta, \eta_{s}=\frac{\partial}{\partial s} \eta .
$$

Let $\mu(s)=k^{\prime}(s)$. Equation (1) transforms into the following system:

$$
\left\{\begin{array}{l}
u_{t t}+\Delta^{2} u-\left(1+k(0) \int_{\Omega}|\nabla u|^{2} d x\right) \Delta u-\int_{0}^{\infty} \mu(s) \Delta \eta(s) d s+a(x) g\left(u_{t}\right)+f(u)=q(x, t)+\kappa \sum_{j=1}^{m} h_{j} \dot{W}_{j} ; \\
\eta_{t}=-\eta_{s}+u_{t} \\
u(t, x)=0, \quad x \in \partial \Gamma, t>0 \\
\eta(x, t, s)=0, \quad x \in \partial \Gamma, t>0, s \in \mathbb{R}^{+} \\
u(\tau, x)=u_{0}(x), u_{t}(\tau, x)=u_{1}(x), x \in \Gamma \\
\eta(x, \tau, s)=\eta_{0}(x, s)=u_{0}(x)-u_{0}(x,-s), x \in \Gamma, s \in \mathbb{R}^{+} .
\end{array}\right.
$$

The following hypotheses are necessary to obtain our main results, infer to [3-5].

(a) The memory kernel $\mu$ is required to satisfy the following hypotheses hold:

$$
\left\{\begin{array}{ll}
\left(\mathbf{H}_{1}\right): & \mu \in \mathbb{C}^{1}\left(\mathbb{R}^{+}\right) \cap \mathbb{L}^{1}\left(\mathbb{R}^{+}\right), \\
\left(\mathbf{H}_{2}\right): & \mu(s) \geq 0, \mu^{\prime}(s) \leq 0, \forall s \in \mathbb{R}^{+}, \\
\left(\mathbf{H}_{3}\right): & \mu^{\prime}(s)+k_{1} \mu(s) \leq 0, \forall s \in \mathbb{R}^{+} \text {and } \sigma>0, \\
\left(\mathbf{H}_{4}\right): & m_{0}:=\int_{0}^{\infty} \mu(s) d s<\infty .
\end{array} .\right.
$$

(b) We need the following condition on $q(x, t) \in \mathrm{七}_{l o c}^{2}\left(\mathbb{R}, L^{2}(\Gamma)\right)$, there exists a positive constant $\sigma$ satisfy that

$$
\begin{cases}\left(\mathbf{Q}_{1}\right): & \int_{-\infty}^{\tau} e^{\sigma r}\|q(\cdot, r)\|^{2} d r<\infty, \forall r \in \mathbb{R}, \\ \left(\mathbf{Q}_{2}\right): & \|q(x, t)\|^{2}=\sup _{r \in \mathbb{R}} \int \| q\left(., r \|^{2} d s<\infty \forall r \in \mathbb{R},\right. \\ \left(\mathbf{Q}_{3}\right): & \lim _{k \rightarrow \infty} \int_{-\infty}^{\tau} \int_{|x| \geq k} e^{\sigma r}|g(x, r)|^{2} d x d r=0, \forall \tau \in \mathbb{R} .\end{cases}
$$

The basic concepts and notions of random attractors for the infinite dimensional was recently presented by in [6-9]. A random attractor of RDS is a measurable and compact invariant random set attracting all orbits. whilst such an attracting set exists, it is the smallest attracting compact set and the largest invariant set. In recent years, a random attractor for autonomous and non-autonomous stochastic dynamical systems have been studied by many authors, see for example [10-16] and the references therein.

In the deterministic case; that is, $\kappa=0$ in (1), the asymptotic behavior of the solution for global attractors an extensible beam equation with localized nonlinear damping with memory has been studied in [5,17-19].

In [20], for the case of $\mu=0$ in (1), the authors investigated the existence of random attractor for the stochastic an extensible beam equation with localized nonlinear damping without memory. But, there were no results even for the bounded case. While it is far just our interest in this paper. To the best of our knowledge, the dynamics of system (1) involving but essential difficulties in showing compactness by using the uniform estimates on the tails of solution. Motivated by a similar technique of [16].

The rest of the paper is organized as follows. In Section 2, we recall some basic concepts and properties for general random dynamical systems. In Section 3, we first provide some basic settings about (1) and show that it generates a random dynamical system in proper function space. In Section 4, we prove the existence of a unique random attractor of the random dynamical system by bounded absorbing set and using a compact measurable pullback attracting set. 


\section{Preliminaries}

In this section, we recall some basic concepts related to random attractors for stochastic dynamical systems. The readers are referred to [6-8] for more details. Which are crucial for getting our main results. Let $(\Omega, \mathcal{F}, P)$ be a probability space and $(X, d)$ be a Polish space with the Borel $\sigma$-algebra $B(X)$. The distance between $x \in X$ and $B \subseteq X$ is denoted by $d(x, B)$. If $B \subseteq X$ and $C \subseteq X$, the Hausdorff semi-distance from $B$ to $C$ is denoted by way of $d(B, C)=\sup _{x \in B} d(x, C)$.

Definition 1. $\left(\Omega, \mathcal{F}, P,\left(\theta_{t}\right)_{t \in \mathbb{R}}\right)$ is called a metric dynamical system if $\theta: \mathbb{R} \times \Omega \longrightarrow \Omega$ is $(\mathcal{B}(\mathbb{R}) \times$ $\mathcal{F}, \mathcal{F}$ )-measurable, $\theta_{0}$ is the identity on $\Omega, \theta_{s+t}=\theta_{t} \circ \theta_{s}$ for all $\mathrm{s}, \mathrm{t} \in \mathbb{R}$ and $\theta_{0} \mathrm{P}=\mathrm{P}$ for all $\mathrm{t} \in \mathbb{R}$.

Definition 2. A mapping $\Phi(t, \tau, \omega, x): \mathbb{R}^{+} \times \mathbb{R} \times \Omega \times X \rightarrow X$ is called continuous cocycle on $X$ over $\mathbb{R}$ and $\left(\Omega, \mathcal{F}, P,\left(\theta_{t}\right)_{t \in \mathbb{R}}\right)$, if for all $\tau \in \mathbb{R}, \omega \in \Omega$ and $t, s \in \mathbb{R}^{+}$, the following conditions are satisfied:

i) $\Phi(t, \tau, \omega, x): \mathbb{R}^{+} \times \mathbb{R} \times \Omega \times X \rightarrow X$ is a $\left(\mathcal{B}\left(\mathbb{R}^{+}\right) \times \mathcal{F}, \mathcal{B}(\mathbb{R})\right)$ measurable mapping,

ii) $\Phi(0, \tau, \omega, x)$ is identity on $X$,

iii) $\Phi(t+s, \tau, \omega, x)=\Phi\left(t, \tau+s, \theta_{s} \omega,, x\right) \circ \Phi(s, \tau, \omega, x)$,

iv) $\Phi(t, \tau, \omega, x): X \rightarrow X$ is continuous.

Definition 3. Let $2^{X}$ be the collection of all subsets of $X$, a set valued mapping $(\tau, \omega) \mapsto \mathcal{D}(t \omega): \mathbb{R} \times \Omega \mapsto 2^{X}$ is called measurable with respect to $\mathcal{F}$ in $\Omega$ if $\mathcal{D}(t \omega)$ is a (usually closed) nonempty subset of $X$ and the mapping $\omega \in \Omega \mapsto d(X, B(\tau, \omega))$ is $(\mathcal{F}, \mathcal{B}(\mathbb{R}))$-measurable for every fixed $x \in X$ and $\tau \in \mathbb{R}$. Let $B=$ $B(t, \omega) \in \mathcal{D}(t, \omega): \tau \in \mathbb{R}, \omega \in \Omega$ is called a random set.

Definition 4. A random bounded set $B=\{B(\tau, \omega): \tau \in \mathbb{R}, \omega \in \Omega\} \in \mathcal{D}$ of $X$ is called tempered with respect to $\{\theta(t)\}_{t \in \Omega}$, if for p-a.e $\omega \in \Omega$,

$$
\lim _{t \mapsto \infty} e^{-\beta t} d\left(B\left(\theta_{-t} \omega\right)\right)=0, \forall \beta>0
$$

where $d(B)=\sup _{x \in B}\|x\|_{X}$.

Definition 5. Let $\mathcal{D}$ be a collection of random subset of $X$ and $K=\{K(\tau, \omega): \tau \in \mathbb{R}, \omega \in \Omega\} \in \mathcal{D}$, then $K$ is called an absorbing set of $\Phi \in \mathcal{D}$ if for all $\tau \in \mathbb{R}, \omega \in \Omega$ and $B \in \mathcal{D}$, there exists, $T=T(\tau, \omega, B)>0$ such that

$$
\Phi\left(t, \tau, \theta_{-t} \omega, B\left(\tau, \theta_{-t} \omega\right)\right) \subseteq K(\tau, \omega), \forall t \geq T
$$

Definition 6. Let $\mathcal{D}$ be a collection of random subset of $X$, the $\Phi$ is said to be $\mathcal{D}$-pullback asymptotically compact in $X$ if for p-a.e $\omega \in \Omega,\left\{\Phi\left(t_{n}, \theta_{-t_{n}} \omega, x_{n}\right)\right\}_{n=1}^{\infty}$ has a convergent subsequence in $X$ when $t_{n} \mapsto \infty$ and $x_{n} \in B\left(\theta_{-t_{n}} \omega\right)$ with $\{B(\omega)\}_{\omega \in \Omega} \in \mathcal{D}$.

Definition 7. Let $\mathcal{D}$ be a collection of random subset of $X$ and $\mathcal{A}=\{\mathcal{A}(\tau, \omega): \tau \in \mathbb{R}, \omega \in \Omega\} \in \mathcal{D}$, then $\mathcal{A}$ is called a $\mathcal{D}$-random attractor (or $\mathcal{D}$-pullback attractor) for $\Phi$, if the following conditions are satisfied for all $t \in \mathbb{R}^{+}, \tau \in \mathbb{R}$ and $\omega \in \Omega$

i) $\mathcal{A}(\tau, \omega)$ is compact, and $\omega \mapsto d(x, \mathcal{A}(\omega))$ is measurable for every $x \in X$,

ii) $\mathcal{A}(\tau, \omega)$ is invariant, that is $\Phi(t, \tau, \omega, \mathcal{A}(\tau, \omega))=\mathcal{A}\left(\tau+t, \theta_{t} \omega\right), \forall t \geq \tau$,

iii) $\mathcal{A}(\tau, \omega)$ attracts every set in $\mathcal{D}$, that is for every $B=\{B(\tau, \omega): \tau \in \mathbb{R}, \omega \in \Omega\} \in \mathcal{D}$, $\lim _{t \mapsto \infty} d_{X}\left(\Phi\left(t, \tau, \theta_{-t} \omega, B\left(\tau, \theta_{-t} \omega\right)\right), \mathcal{A}(\tau, \omega)\right)=0$, where $d_{X}$ is the Hausdorff semi-distance given by $d_{X}(Y, Z)=\sup _{y \in Y} \inf _{z \in Z}\|y-z\|_{X}$ for any $Y \in X$ and $Z \in X$.

Lemma 1. Let $\mathcal{D}$ be a neighborhood-closed collection of $(\tau, \omega)$-parameterized families of nonempty subsets of $X$ and $\Phi$ be a continuous cocycle on $X$ over $\mathbb{R}$ and $\left(\Omega, \digamma, P,\left(\theta_{t}\right)_{t \in \mathbb{R}}\right)$. Then $\Phi$ has a pullback $\mathcal{D}$-attractor $\mathcal{A}$ in $\mathcal{D}$ if and only if $\Phi$ is pullback $\mathcal{D}$-asymptotically compact in $X$ and $\Phi$ has a closed, $\digamma$-measurable pullback $\mathcal{D}$-absorbing set $K \in \mathcal{D}$, the unique pullback $\mathcal{D}$-attractor $\mathcal{A}=\mathcal{A}(\tau, \omega)$ is given

$$
\left.\mathcal{A}(\tau, \omega)=\bigcap_{r \geq 0} \overline{\bigcup_{t \geq r} \Phi\left(t, \tau-t, \theta_{-t} \omega, K\left(\tau-t, \theta_{-t} \omega\right)\right.}\right) \tau \in \mathbb{R}, \omega \in \Omega .
$$




\section{Existence and uniqueness of solution}

In this Section, first, we collect some important results that will help to achieve our goal. Let $A=$ $\Delta^{2}, A^{\frac{1}{2}}=-\Delta$ and $D(A)=\left\{u \in H^{4}: \Delta \in H_{0}^{1}\right.$. We can define the powers $A^{v}$ is Hilbert space and a norm hold $D\left(A^{\frac{v}{4}}\right)=\mathbf{V}_{v}=\left\|A^{\frac{v}{4}} u\right\|^{2}, v \in \mathbb{R}$. Especially, $\mathbf{V}_{0} \hookrightarrow L^{2}$ and $\mathbf{V}_{1} \hookrightarrow H^{2} \cap H_{0}^{1}$. We denote that the injection $\mathbf{V}_{v_{1}} \hookrightarrow \mathbf{V}_{v_{2}}$ is compact embeddings, if $v_{1}>v_{2}$ in conjunction with the generalized Poincaré inequality;

$$
\|u\|_{v+1}^{4} \geq \lambda_{1}\|u\|_{v}^{4}
$$

where $\lambda_{1}$ is the first eigenvalue of $A$. Additionally we outline the subsequent

$$
\left\{\begin{array}{l}
(u, v)=\int_{\Gamma} u v d x=\|u\|\|v\|, \\
(u, u)=\|u\|^{2} \\
((u, v))=\int_{\Gamma} \triangle u \triangle v d x=\|\Delta u\|\|\triangle v\|, \\
((u, u))=\int_{\Gamma} \triangle u \triangle u d x=\|\Delta u\|^{2} .
\end{array}\right.
$$

Much like [18], for the memory kernel hypotheses $\mu(\cdot)$, we suppose $L_{\mu}^{2}\left(\mathbb{R}^{+} ; \mathbf{V}_{v}\right)$ the Hilbert space of function $\eta: \mathbb{R}^{+} \longrightarrow \mathbf{V}_{v}$ endowed with the inner product and norm respectively,

$$
\left\{\begin{array}{l}
(u, v)_{\mu, v}=\int_{0}^{\infty} \mu(s)\left(A^{\frac{v}{4}} u(s), A^{\frac{v}{4}} v(s)\right) d s, \\
\left(\eta_{1}, \eta_{2}\right)_{\mu, v}=\int_{0}^{\infty} \mu(s)\left(A^{\frac{v}{4}} \eta_{1}(s), A^{\frac{v}{4}} \eta_{2}(s)\right) d s \\
\|\eta\|_{\mu, v}^{2}=\left(A^{\frac{v}{4}} \eta, A^{\frac{v}{4}} \eta\right)_{\mu}=\int_{0}^{\infty} \mu(s)\|\eta\|_{v}^{2} d s
\end{array}\right.
$$

specially, $\|u\|_{\mu, v}^{2}=\|u\|_{\mu, 1}^{2}$. Let, we define the product Hilbert space $E=\mathbf{V}_{0} \times \mathbf{V}_{1} \times L_{\mu}^{2}\left(\mathbb{R}^{+} ; \mathbf{V}_{1}\right)$.

To convert the version of Problem (6) with a random perturbation term right into a deterministic one with a random parameter $\omega$, we introduce an Ornstein-Uhlenbeck process driven by means of the Brownian motion, which satisfies the subsequent differential equation

$$
d z_{j}+\delta z_{j} d t=d W_{j}(t)
$$

Its unique stationary solution is given by

$$
z_{j}\left(\theta_{t} \omega_{j}\right)=-\delta \int_{-\infty}^{0} e^{\delta s}\left(\theta_{t} \omega_{j}\right)(s) d s, s \in \mathbb{R}, t \in \mathbb{R}, \omega_{j} \in \Omega
$$

From [6,16], it is recognized that the random variable $\left|z_{j}\left(\omega_{j}\right)\right|$ is tempered and there is an invariant set $\bar{\Omega} \subseteq \Omega$ of full $P$ measure such that $z_{j}\left(\theta_{t} \omega_{j}\right)=z_{j}\left(t, \omega_{j}\right)$ is continuous in $t$, for each $\omega \in \bar{\Omega}$. For comfort, we shall write $\bar{\Omega}$ as $\Omega$. It follows from Proposition 3.4 in [16], that for any $\epsilon>0$, there exists a tempered characteristic $\curlyvee(\omega)>0$ such that

$$
\sum_{j=1}^{m}\left(\left|z_{j}\left(\omega_{j}\right)\right|^{2}+\left|z_{j}\left(\omega_{j}\right)\right|^{\gamma+2}\right) \leq \curlyvee(\omega),
$$

where $\curlyvee(\omega)$ satisfies for, p-a.e. $\omega \in \Omega$,

$$
\curlyvee\left(\theta_{t} \omega\right) \leq e^{\varepsilon|t|} \curlyvee(\omega), t \in \mathbb{R}
$$

Then, it follows from the above inequality, for p-a.e. $\omega \in \Omega$,

$$
\sum_{j=1}^{m}\left(\left|z_{j}\left(\theta_{t} \omega_{j}\right)\right|^{2}+\left|z_{j}\left(\theta_{t} \omega_{j}\right)\right|^{\gamma+2}\right) \leq e^{\varepsilon|t|} \curlyvee(\omega), t \in \mathbb{R}
$$

Put $\kappa h(x) z\left(\theta_{t} \omega\right)=\kappa \sum_{j=1}^{m} h_{j} z_{j}\left(\theta_{t} \omega_{j}\right)$, which solves $d z+\delta z d t=\sum_{j=1}^{m} h_{j} \dot{W}_{j}(t)$.

Let $v(t, \tau, x, \omega)=u_{t}+\varepsilon u-\kappa h(x) z\left(\theta_{t} \omega\right)$, we handy to reduce (6) to an evolution equation of the first-order in time random partial differential equation (RPDE): 


$$
\left\{\begin{array}{l}
u_{t}=v-\varepsilon u+\kappa h(x) z\left(\theta_{t} \omega\right) \\
v_{t}-\varepsilon v+\varepsilon^{2} u+A u+\int_{0}^{\infty} \mu(s) A^{\frac{1}{2}} \eta(s) d s=\left(1+k(0) \int_{\Omega}|\nabla u|^{2} d x\right) A^{\frac{1}{2}} u-a(x) g\left(u_{t}\right)-f(u)+g(x, t)+\varepsilon \kappa h(x) z\left(\theta_{t} \omega\right) \\
\eta_{t}+\eta_{s}=-\varepsilon u+v+\kappa h(x) z\left(\theta_{t} \omega\right) \\
u(x, \tau)=u_{0}(x), u_{t}(\tau, x)=u_{1}(x), x \in \Gamma \\
v(x, \tau)=v_{0}(x)=u_{1}(x)+\varepsilon u_{0}(x)-\kappa h(x) z\left(\theta_{t} \omega\right) \\
\eta(x, \tau, s)=\eta_{0}=u_{0}(x)-u_{0}(x,-s), x \in \Gamma, s \in \mathbb{R}^{+}
\end{array}\right.
$$

Consequently the stochastic system for the system (16) becomes

$$
\left\{\begin{array}{l}
\psi^{\prime}+H(\psi)=Q(\psi, t, \omega) \\
\psi(\tau, \omega)=\left(u_{0}(x), u_{1}(x)+\varepsilon u_{0}(x)-\kappa h(x) z\left(\theta_{t} \omega\right), \eta_{0}\right)^{\top}, \psi=(u, v, \eta)^{\top}
\end{array}\right.
$$

in which $\psi=\left(\begin{array}{c}u \\ v \\ \eta\end{array}\right), \quad H(\psi)=\left(\begin{array}{c}\varepsilon u-v \\ -\varepsilon v+\varepsilon^{2} u+A u+\int_{0}^{\infty} \mu(s) A^{\frac{1}{2}} \eta(s) d s \\ \varepsilon u-v+\eta_{s}\end{array}\right)$ and $Q(\psi, \omega, t)=$

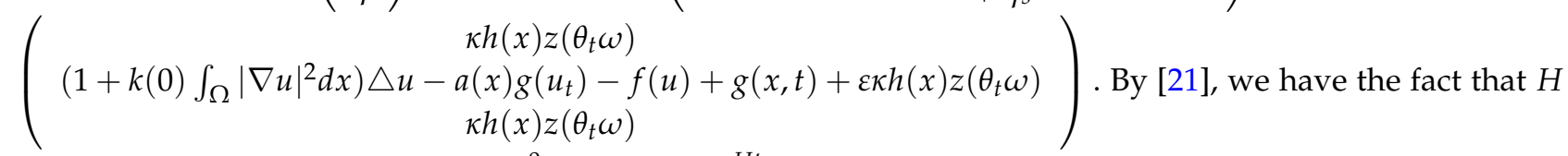
is the infinitesimal generators of $\mathbf{C}^{0}$-semigroup $e^{H t}$ on $E(\Gamma)$. It is not difficult to check that the function $Q(\psi, \omega, t): E \rightarrow E$ is locally Lipschitz continuous with respect to $\psi$ and bounded for each $\omega \in \Omega$.

In order to obtain the random attractor of the Problem (17) has a unique solution in the mild sense, by the classical semigroup theory of existence and uniqueness of solutions of evolution differential equations [21], we get the following result.

Theorem 1. Let (2)-(5) and (7)-(8) hold. Then, for every $\tau \in \mathbb{R}, \omega \in \Omega$ and $\chi_{\tau} \in E(\Gamma)$, the Problem (17) has a unique solution $\chi\left(t, \tau, \omega, \chi_{\tau}\right)$ which is continuous with respect to $\left(u_{0}, v_{0}, \eta_{0}\right)^{\top} \in E(\Gamma)$ such that $\chi_{\tau}$ and $\chi\left(t, \tau, \omega, \chi_{\tau}\right)$ satisfies the integral equation

$$
\chi\left(t, \tau, \omega, \chi_{\tau}\right)=e^{-H(t)} \chi_{\tau}(\omega)+\int_{0}^{t} e^{-H(t-r)} Q(\chi, r, \omega) d r .
$$

Moreover, $\chi\left(t, \tau, \omega, \chi_{\tau}\right)$ is continuous in $\chi_{\tau}$ and measurable in $\omega$.

Theorem 2. Let (2)-(4) and (7)-(8) hold. Then, for any $\tau \in \mathbb{R}, \omega \in \Omega$ and $\chi_{\tau} \in E(\Gamma)$, such that $\chi\left(t, \tau, \omega, \chi_{\tau}\right) \in$ $E(\Gamma)$ is a solution of the Problem (17) satisfy the properties of continuous random dynamical system over $\mathbb{R}$ and $\left(\Omega, \mathcal{F}, P,\left(\theta_{t}\right)_{t \in \mathbb{R}}\right)$. We can show that for P-a.s. every $\omega \in \Omega$, for all $\mathrm{T}>0$

(1) if $\chi_{\tau}(\omega) \in E$, then $\chi\left(\mathrm{T}, \omega, \chi_{\tau}\right)=\chi\left(\mathrm{T}, \omega, \chi_{\tau}\right) \in C([t, t+\mathrm{T})$; E),

(2) $\chi\left(t, \tau, \omega, \chi_{\tau}\right)$ is jointly continuous into t and measurable in $\chi_{\tau}(\omega)$,

(3) the solution mapping of (18) holds the properties of continuous cocycle.

From the Theorem 1, we can define a continuous random dynamical system over $\mathbb{R}$ and $\left(\Omega, \mathcal{F}, P,\left(\theta_{t}\right)_{t \in \mathbb{R}}\right)$, that is, $\boldsymbol{\Phi}\left(t, \tau, \omega, \chi_{\tau}\right): \mathbb{R} \times \mathbb{R}^{+} \times \Omega \times E \mapsto E, t \geq \tau$, such that

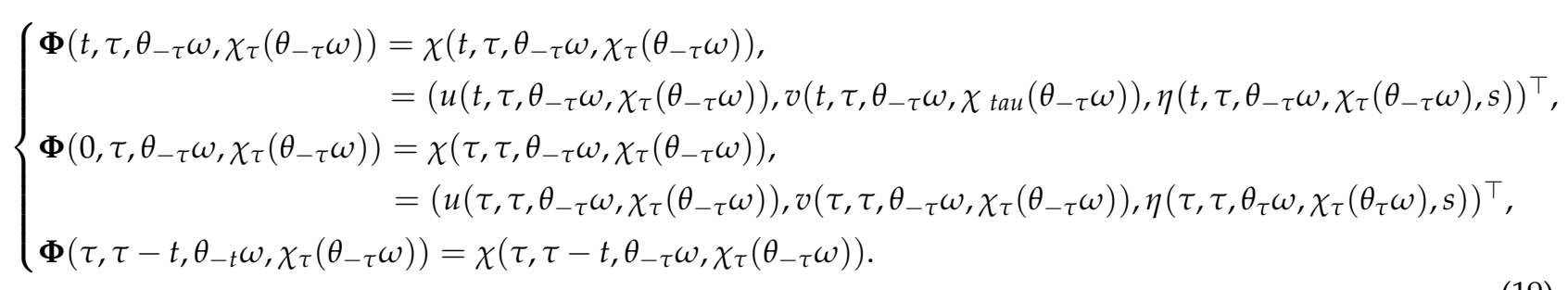

It generates a random dynamical system. Moreover

$$
\hat{\boldsymbol{\Phi}}\left(\tau, \tau, \theta_{-\tau} \omega, \chi_{\tau}\left(\theta_{-\tau} \omega\right): \chi\left(\tau, \tau, \theta_{-\tau} \omega, \chi_{\tau}\left(\theta_{-\tau} \omega\right)+\left(0, \kappa h(x) z\left(\theta_{\tau} \omega\right), 0\right)^{\top} \mapsto \varphi(t, \omega)+\left(0, \kappa h(x) z\left(\theta_{\tau} \omega\right), 0\right)^{\top} .\right.\right.
$$


To show the conjugation of the solution for the stochastic partial differential Equation (17) and the random partial differential Equation (19), introducing the homeomorphism $P\left(\theta_{t} \omega\right)(y, w, \zeta(s))^{\top}=(y, w-$ $\left.\varepsilon y+\kappa h(x) z\left(\theta_{t} \omega\right), \zeta(s)\right)^{\top},(y, w, \zeta(s))^{\top} \in E(\Gamma)$ with an inverse homeomorphism $P^{-1}\left(\theta_{t} \omega\right)(y, w, \zeta(s))^{\top}=$ $\left(y, w+\varepsilon y-\kappa z\left(\theta_{t} \omega\right), \zeta(s)\right)^{\top}$, then we have the transformation

$$
\hat{\boldsymbol{\Phi}}(\tau, t, \omega)=P\left(\theta_{t} \omega\right) \boldsymbol{\Phi}(t, \omega) P^{-1}\left(\theta_{t} \omega\right), E \mapsto E, t \geq \tau .
$$

Consider the equivalent RDS and introduce the isomorphism and has the inverse isomorphism:

$$
\left\{\begin{array}{l}
\breve{\boldsymbol{\Phi}}(\tau, t, \omega)=T_{\varepsilon} \hat{\boldsymbol{\Phi}}(t, \omega) T_{-\varepsilon}: \chi_{\tau} \mapsto \varphi\left(t+\tau, \tau, \theta_{-\tau} \omega, \chi_{\tau}\left(\theta_{-\tau} \omega\right)\right) \\
\varphi^{\prime}+H(\varphi)=\bar{Q}(\varphi, t, \omega) \\
\varphi(\tau, \omega)=\varphi_{\tau}=\left(u_{0}(x), y_{1}(x)-\varepsilon y_{0}(x), \eta_{0}\right)^{\top}
\end{array}\right.
$$

where

$$
\begin{gathered}
\varphi=(y, w, \eta)^{\top}=\left(y, y_{t}+\varepsilon y, \eta\right)^{\top}, \\
T_{\varepsilon} \varphi=(y, w, \eta)^{\top}=(y, w+\varepsilon y, \eta)^{\top}, \\
T_{-\varepsilon} \varphi=(y, w, \eta)^{\top}=(y, w-\varepsilon y, \eta)^{\top}, \\
H(\varphi)=\left(\begin{array}{c}
\varepsilon y-w \\
-\varepsilon w+\varepsilon^{2} y+A y+\int_{0}^{\infty} \mu(s) A^{\frac{1}{2}} \eta(s) d s \\
\varepsilon y-w+\eta_{s}
\end{array}\right),
\end{gathered}
$$

and

$$
\bar{Q}(\varphi, \omega, t)=\left(\begin{array}{c}
0 \\
\left(1+k(0) \int_{\Omega}|\nabla y|^{2} d x\right) \Delta y-a(x) g\left(y_{t}\right)-f(y)+g(x, t)+\kappa h(x) z\left(\theta_{t} \omega\right) \\
0
\end{array}\right)
$$

is also a random dynamical systems corresponding to the Equation (17). Therefore, $\boldsymbol{\Phi}, \hat{\boldsymbol{\Phi}}$ and $\check{\boldsymbol{\Phi}}$ are equivalent to each other in dynamics.

\section{Random absorbing set}

In this section, we will show boundedness of the solutions for Equation (17). The existence of a pullback absorbing set $\boldsymbol{\Phi} \in \mathcal{D}$ and the asymptotic compactness of the random dynamical system associated with the Equation (17). We always assume that $\mathcal{D}$ is the collection of all tempered subsets of $E(\Gamma)$ from now on.

Lemma 2. Let (2)-(4) and (7)-(8) hold. Then, for any $\tau \in \mathbb{R}, \omega \in \Omega$ and $\chi_{\tau-t} \in E(\Gamma)$, there exists a random ball $\{K(\omega)\}_{\omega \in \Omega} \in \mathcal{D}$ centered at 0 with random radius $M(\omega) \geq 0$ such that $\{K(\omega)\}$ is a random absorbing set for $\Phi$ in $\mathcal{D}$, that is, for any $B=\{B(\omega)\}_{\omega \in \Omega} \in \mathcal{D}, P$-almost surely, there exists a $T=T(\tau, \omega, B)>0$ and $\chi_{\tau-t}(\omega) \in B(\omega)$ such that

$$
\left\|\chi\left(r, \tau-t, \theta_{-\tau} \omega, \chi_{\tau-t}\right)\right\|_{E}^{2} \leq M_{0}^{2}(\omega)
$$

where $M_{0}(\omega)$ is a positive random function, that is

$$
\Phi\left(t, \tau, \theta_{-t} \omega, B\left(\tau, \theta_{-t} \omega\right)\right) \subseteq K(\tau, \omega) \text { for all } t \geq T .
$$

Proof. Taking the inner product of the first term of (23) with $\chi=(u, v, \eta) \in E, \quad v=\frac{d u}{d t}+\varepsilon u-\kappa h(x) z\left(\theta_{t} \omega\right)$, we find that

$$
\left(\chi^{\prime}, \chi\right)+(H(\chi), \chi)=(\mathbf{F}(t, x, \chi), \chi)
$$

Using Hölder, Young and Poincarè inequalities and after simple computation, we gain

$$
\begin{aligned}
(H(\chi), \chi) & =\left(\begin{array}{c}
\varepsilon u-v \\
-\varepsilon v+\varepsilon^{2} u+A u+\int_{0}^{\infty} \mu(s) A^{\frac{1}{2}} \eta(s) d s \\
\varepsilon u-v+\eta_{s}
\end{array}\right)\left(\begin{array}{c}
u \\
v \\
\eta
\end{array}\right) \\
& =\varepsilon\|\Delta u\|^{2}+\varepsilon^{2}(u, v)-\varepsilon\|v\|^{2}+\left(\varepsilon u+\eta_{s}, \eta\right)
\end{aligned}
$$




$$
\begin{aligned}
& =\varepsilon\|\Delta u\|^{2}+\varepsilon^{2}(u, v)-\varepsilon\|v\|^{2}-\frac{\delta}{4}\|\nabla \eta\|_{\mu}^{2}-\frac{m_{0} \varepsilon^{2}}{2 \lambda}\|\Delta u\|^{2}+\frac{\delta}{2}\|\nabla \eta\|_{\mu}^{2} \\
& =\varepsilon\|\Delta u\|^{2}+\varepsilon^{2}(u, v)-\varepsilon\|v\|^{2}-\frac{m_{0} \varepsilon^{2}}{2}\|\nabla u\|^{2}+\frac{\delta}{4}\|\nabla \eta\|_{\mu}^{2} .
\end{aligned}
$$

Using Cauchy-Schwartz inequality and Young inequality, we obtain

$$
(\mathbf{F}(t, x, \chi), \chi)=\left(\begin{array}{c}
k h(x) z\left(\theta_{t} \omega\right) \\
\left(1+k(0) \int_{\Omega}|\nabla y|^{2} d x\right) A^{\frac{1}{2}} u-a(x) g\left(u_{t}\right)-f(u)+g(x, t)+\kappa h(x) z\left(\theta_{t} \omega\right) \\
k h(x) z\left(\theta_{t} \omega\right)
\end{array}\right)\left(\begin{array}{c}
u \\
v \\
\eta
\end{array}\right)
$$

From (3) $\left(\mathbf{A}_{2}\right),\left(\mathbf{A}_{4}\right)$, we obtain

$$
\begin{aligned}
\left.-\left(1+k(0)\|\nabla u\|^{2}\right) \triangle u, v\right) & =-\left(\left(1+k(0)\|\nabla u\|^{2}\right) \nabla, \nabla\left(\frac{d u}{d t}+\varepsilon u-a h(x) z\left(\theta_{t} \omega\right)\right)\right. \\
& \left.\leq-\left(1+k(0)\|\nabla u\|^{2}\right)\left(\frac{1}{2} \frac{d}{d t}\|\nabla u\|^{2}+\frac{\varepsilon}{2}\|\nabla u\|^{2}\right)+\frac{\kappa^{2}}{2 \varepsilon} \| \nabla h(x)\right) \|^{2}\left|z\left(\theta_{t} \omega\right)\right|^{2},
\end{aligned}
$$

and from (2) and (4), it is easy to show that

$$
\begin{aligned}
\left(a(x) g\left(u_{t}\right), v\right) & =\left(\alpha_{0} g(\vartheta)\left(v-\varepsilon u+\kappa h(x) z\left(\theta_{t} \omega\right)-g(0)\right), v\right) \\
& \leq \alpha_{0} \alpha_{1}\|v\|^{2}+\alpha_{0}\left(-\alpha_{2} \varepsilon u+g^{\prime}(\vartheta) \kappa h(x) z\left(\theta_{t} \omega, v\right)\right.
\end{aligned}
$$

where $\vartheta$ is between 0 and $v-\varepsilon u+\kappa h(x) z\left(\theta_{t} \omega\right)$.

$$
\begin{aligned}
& (q(x, t), v)=\|q(x, t)\|\|v\| \leq \frac{\|q(x, t)\|^{2}}{2\left(\alpha_{0} \alpha_{1}-\varepsilon\right)}+\frac{\alpha_{0} \alpha_{1}-\varepsilon}{2}\|v\|^{2}, \\
& \left(\left(k h(x) z\left(\theta_{t} \omega\right), u\right)\right) \leq\|\Delta u\|\|\Delta h(x)\|\left|z\left(\theta_{t} \omega\right)\right| \leq \frac{\varepsilon}{4}\|\Delta u\|^{2}+\frac{k^{2}}{\varepsilon}\|\Delta h(x)\|^{2}\left|z\left(\theta_{t} \omega\right)\right|^{2}, \\
& \left(k h(x) z\left(\theta_{t} \omega\right), \eta\right)_{\mu} \leq \frac{m_{0} k^{2}}{\delta}\|\nabla h(x)\|^{2}\left|z\left(\theta_{t} \omega\right)\right|^{2}+\frac{\delta}{4}\|\nabla \eta\|_{\mu}^{2} \\
& \left.\left(\alpha_{0} g^{\prime}(\vartheta)-2 \varepsilon\right) \kappa h(x) z\left(\theta_{t} \omega\right), v\right) \leq \frac{2 \alpha_{2}^{2} \kappa^{2}}{\alpha_{0} \alpha_{1}-\varepsilon}\|h(x)\|^{2}\left|z\left(\theta_{t} \omega\right)\right|^{2}+\frac{\alpha_{0} \alpha_{1}-\varepsilon}{8}\|v\|^{2} .
\end{aligned}
$$

By second term for right hand side of (26) and (29), we can get

$$
\varepsilon\left(\varepsilon-\alpha_{2} \alpha_{0}\right)(u, v) \geq-\frac{\alpha_{0} \alpha_{2} \varepsilon}{\lambda}\|\nabla u\|\|v\| \geq \frac{2\left(\alpha_{0}^{2} \alpha_{2}^{2} \varepsilon^{2}\right)}{\left(\alpha_{0} \alpha_{1}-\varepsilon\right) \lambda^{2}}\|\nabla u\|^{2}-\frac{\alpha_{0} \alpha_{1}-\varepsilon}{8}\|v\|^{2} .
$$

About the nonlinearity, by (4), Hölder inequality and the Sobolev embedding theorem, we estimate that

$$
(f(u), v)=\left(f(u), \frac{d u}{d t}+\varepsilon u-\kappa h(x) z\left(\theta_{r-\tau} \omega\right)\right) \geq \frac{d}{d t} F(u)+\varepsilon C_{3}(F(u)-|\Gamma|)+\left(f(u), \kappa h(x) z\left(\theta_{r-\tau} \omega\right)\right) .
$$

From $(3)_{\left(\mathbf{A}_{3}\right)-\left(\mathbf{A}_{5}\right)}$, we have

$$
\begin{aligned}
& \left(f(u), \kappa h(x) z\left(\theta_{r-\tau} \omega\right)\right) \leq C_{1} \int_{\Gamma}\left(1+|u|^{\gamma}\right) \kappa h(x) z\left(\theta_{r-\tau} \omega\right) d x \\
& \leq C_{1} \kappa\|h(x)\|\left|z\left(\theta_{r-\tau} \omega\right)\right|+C_{1} \kappa \int_{\Gamma}\left(|u|^{\gamma+1}\right)^{\frac{\gamma}{\gamma+1}}\|h(x)\|_{L^{\gamma+1}(U)}\left|z\left(\theta_{r-\tau} \omega\right)\right|^{\frac{\gamma}{\gamma+1}} \\
& \leq C_{1} \kappa\|h(x)\|\left|z\left(\theta_{r-\tau} \omega\right)\right|+C_{1} \kappa\left(\frac{1}{C_{2}} F(u)+\int_{\Gamma} d x\right)^{\frac{\gamma}{\gamma+1}}\|h(x)\|_{L^{\gamma+1}(\Gamma)}\left|z\left(\theta_{r-\tau} \omega\right)\right|^{\frac{\gamma}{\gamma+1}} \\
& \leq C_{1} \kappa\|h(x)\|\left|z\left(\theta_{r-\tau} \omega\right)\right|+\frac{\kappa \varepsilon C_{1}}{2}|\Gamma|+\frac{\varepsilon C_{1}}{2 C_{2}} F(u)+C_{1}^{\frac{\gamma}{\gamma+1}} \mathcal{K}^{\frac{\gamma}{\gamma+1}}\|h(x)\|_{H_{0}^{1}(\Gamma)}^{\gamma+1}\left|z\left(\theta_{r-\tau} \omega\right)\right|^{\gamma+1} .
\end{aligned}
$$


Inserting the above two inequalities together, it yields that

$$
\begin{aligned}
& (f(u), v)= \\
& \frac{d}{d t} F(u)+\frac{\varepsilon\left(2 C_{3}-C_{1} C_{2}^{-1}\right)}{2}(F(u)-|\Gamma|)+C_{1} \kappa|| h(x)\left\|\left|z\left(\theta_{r-\tau} \omega\right)\right|+C_{1}^{\frac{\gamma}{\gamma+1}} \kappa^{\gamma+1}\right\| h(x) \|_{H_{0}^{1}(\Gamma)}^{\gamma+1}\left|z\left(\theta_{r-\tau} \omega\right)\right|^{\gamma+1},
\end{aligned}
$$

Collecting all inequalities (25)-(37), it leads to

$$
\begin{aligned}
& \frac{d}{d t}\left(\|v\|^{2}+\|\nabla u\|^{2}+\left(1+k(0)\|\nabla u\|^{2}\right)\|\nabla u\|^{2}+\frac{\delta}{4}\|\nabla \eta\|_{\mu}^{2}-\frac{\varepsilon\left(2 C_{3}-C_{1} C_{2}^{-1}\right)}{2} \int_{\Gamma} F(u) d x\right) \\
& \quad+\frac{\alpha_{0} \alpha_{1}-\varepsilon}{2}\|v\|^{2}+2 \varepsilon\left(1+k(0)\|\nabla u\|^{2}\right)\|\nabla u\|^{2}+2 \varepsilon^{2}\left(m-\frac{\alpha_{0}^{2} \alpha_{2}^{2}}{\left(\alpha_{0} \alpha_{1}-\varepsilon\right) \lambda^{2}}\right)\|\nabla u\|^{2}+\frac{\delta}{4}\|\nabla \eta\|_{\mu}^{2} \\
& \leq \frac{\|q(x, t)\|^{2}}{\left(\alpha_{0} \alpha_{1}-\varepsilon\right)}+C_{1} \kappa\|h(x)\|\left|z\left(\theta_{r-\tau} \omega\right)\right|+C_{1}^{\frac{\gamma}{\gamma+1}} \kappa^{\gamma+1}\|h(x)\|_{H_{0}^{1}(\Gamma)}^{\gamma+1}\left|z\left(\theta_{r-\tau} \omega\right)\right|^{\gamma+1} \\
& \quad+\varepsilon k^{2}\|\Delta h(x)\|^{2}\left|z\left(\theta_{r-\tau} \omega\right)\right|^{2}+\frac{2 \alpha_{2}^{2} \kappa^{2}}{\alpha_{0} \alpha_{1}-\varepsilon}\|h(x)\|^{2}\left|z\left(\theta_{r-\tau} \omega\right)\right|^{2}+\frac{m_{0} k^{2}}{\delta}\|\nabla h(x)\|^{2}\left|z\left(\theta_{r-\tau} \omega\right)\right|^{2} \\
& \left.\quad+\frac{\kappa^{2}}{2 \varepsilon} \| \nabla h(x)\right) \|^{2}\left|z\left(\theta_{r-\tau} \omega\right)\right|^{2}+\frac{\varepsilon\left(2 C_{3}-C_{1} C_{2}^{-1}\right)}{2}|\Gamma| .
\end{aligned}
$$

Thus

$$
\|\varphi\|_{E(\Gamma)}^{2}=\|v\|^{2}+\|\nabla u\|^{2}+\left(1+k(0)\|\nabla u\|^{2}\right)\|\nabla u\|^{2}+\frac{\delta}{4}\|\nabla \eta\|_{\mu}^{2}-\frac{\varepsilon\left(2 C_{3}-C_{1} C_{2}^{-1}\right)}{2} \int_{\Gamma} F(u) d x,
$$

and

$$
\begin{aligned}
\varrho\left(\theta_{r-\tau} \omega\right)= & \frac{\|q(x, t)\|^{2}}{\left(\alpha_{0} \alpha_{1}-\varepsilon\right)}+C_{1} \kappa\|h(x)\|\left|z\left(\theta_{r-\tau} \omega\right)\right|+C_{1}^{\frac{\gamma}{\gamma+1}} \kappa^{\gamma+1}\|h(x)\|_{H_{0}^{1}(\Gamma)}^{\gamma+1}\left|z\left(\theta_{r-\tau} \omega\right)\right|^{\gamma+1} \\
& +\varepsilon k^{2}\|\Delta h(x)\|^{2}\left|z\left(\theta_{r-\tau} \omega\right)\right|^{2}+\frac{2 \alpha_{2}^{2} \kappa^{2}}{\alpha_{0} \alpha_{1}-\varepsilon}\|h(x)\|^{2}\left|z\left(\theta_{r-\tau} \omega\right)\right|^{2}+\frac{m_{0} k^{2}}{\delta}\|\nabla h(x)\|^{2}\left|z\left(\theta_{r-\tau} \omega\right)\right|^{2} \\
& \left.+\frac{\kappa^{2}}{2 \varepsilon} \| \nabla h(x)\right) \|^{2}\left|z\left(\theta_{r-\tau} \omega\right)\right|^{2}+\frac{\varepsilon\left(2 C_{3}-C_{1} C_{2}^{-1}\right)}{2}|\Gamma| .
\end{aligned}
$$

Since $\varepsilon \in(0,1)$ be small enough such that $\varepsilon^{2}\left(m-\frac{\alpha_{0}^{2} \alpha_{2}^{2}}{\left(\alpha_{0} \alpha_{1}-\varepsilon\right) \lambda^{2}}\right)>0, \quad \frac{\alpha_{0} \alpha_{1}-\varepsilon}{2}>0, \quad \frac{\varepsilon\left(2 C_{3}-C_{1} C_{2}^{-1}\right)}{2}>0$, we will choose $\sigma=\left(\frac{\alpha_{0} \alpha_{1}-\varepsilon}{2}, 2 \varepsilon^{2}\left(m-\frac{\alpha_{0}^{2} \alpha_{2}^{2}}{\left(\alpha_{0} \alpha_{1}-\varepsilon\right) \lambda^{2}}\right)\right)$ and $\tilde{\sigma}=\min \left\{\sigma, \frac{\varepsilon\left(2 C_{3}-C_{1} C_{2}^{-1}\right)}{2}, \frac{\delta}{4}\right\}$, which gives

$$
\frac{d}{d t}\|\varphi(r)\|_{E}^{2}+\tilde{\sigma}\|\varphi(r)\|_{E}^{2} \leq \varrho\left(\theta_{r-\tau} \omega\right) .
$$

Applying Gronwall's Lemma over $[\tau-t, r]$, we find that for $r \geq \tau-t$,

$$
\left\|\varphi\left(r, \tau-t, \omega, \varphi_{\tau-t}(\omega)\right)\right\|_{E}^{2} \leq e^{-\tilde{\sigma}-t)}\left\|\varphi_{\tau-t}\right\|_{E}^{2}+\int_{\tau-t}^{\tau} \varrho\left(\theta_{\varsigma-\tau} \omega\right) e^{-\tilde{\sigma}(t-\varsigma)} d \zeta .
$$

By replacing $\omega$ by $\theta_{-t} \omega$, we get from (42) such that for all $t \geq 0$

$$
\begin{aligned}
& \left\|\varphi\left(r, \tau-t, \theta_{-\tau} \omega, \varphi_{\tau-t}\left(\theta_{-\tau} \omega\right)\right)\right\|_{E}^{2} \leq\left\|\chi\left(r, \tau-t, \theta_{-\tau} \omega, \chi_{\tau-t}\left(\theta_{-\tau} \omega\right)\right)\right\|_{E}^{2} \\
& \leq e^{-\tilde{\sigma} t}\left\|\chi\left(\tau-t, \tau-t, \theta_{-\tau} \omega, \chi_{\tau-t}\left(\theta_{-\tau} \omega\right)\right)\right\|_{E}^{2}+\int_{\tau-t}^{\tau} \varrho\left(\theta_{\zeta-\tau} \omega\right) e^{\tilde{\sigma}(\tau-\varsigma)} d \varsigma .
\end{aligned}
$$

Since $z\left(\theta_{t} \omega\right)$ is a tempered random variable and $\lim _{t \rightarrow \pm \infty} \frac{z\left(\theta_{t} \omega\right)}{t}=0, \int_{ \pm \infty}^{0} \frac{1}{t} z\left(\theta_{r} \omega\right) d r=0 . \quad$ Thus, there exists $M_{0}(\omega)$ and $T=T(\tau, \omega, B)>0$ such that

$$
\begin{gathered}
\limsup _{t \rightarrow-\infty} e^{-\tilde{\sigma} t}\left\|\chi\left(\tau-t, \theta_{-\tau} \omega\right)\right\|_{E}^{2}=0, \\
\int_{-\infty}^{0} \varrho\left(\theta_{\varsigma-\tau} \omega\right) e^{\tilde{\sigma}((\tau-\varsigma))} d \varsigma<+\infty=M_{0}^{2}(\omega),
\end{gathered}
$$




$$
\left\|\chi\left(r, \tau-t, \theta_{-\tau} \omega, \chi_{\tau-t}\left(\theta_{-\tau} \omega\right)\right)\right\|_{E}^{2} \leq M_{0}^{2}(\omega) .
$$

The proof is completed.

Now we decompose the Equation (6) into two parts and also decompose the nonlinear growth term $f \in C^{1}$ in Equation (3) into two parts $f=f_{1}+f_{2}$, where $f_{1}, f_{2}$ satisfy the following respectively

$$
\left\{\begin{array}{l}
\left(\mathbf{A}_{1}\right): u f_{i}(u) \geq 0 \\
\left(\mathbf{A}_{2}\right):\left|f_{1}^{\prime}(u)\right| \leq \mu_{1}\left(1+|u|^{\frac{4}{n-2}}\right), \forall u \in \mathbb{R}, n \geq 3 \\
\left(\mathbf{A}_{3}\right):\left|f_{2}(u)\right| \leq \mu_{2}\left(1+|u|^{\gamma}\right), \forall u \in \mathbb{R}, \\
\left(\mathbf{A}_{4}\right): F_{i}(u)=\int_{0}^{u} f_{i}(r) d r \\
\left(\mathbf{A}_{5}\right): u f_{i}(u) \geq \mu_{i}(F(u)-1), \\
\left(\mathbf{A}_{6}\right): k_{0}\left(|u|^{\gamma+1}-1\right) \leq F_{i}(u) \leq k_{1} u f_{i}(u)+C_{\mu}
\end{array}\right.
$$

where $\mu_{i}, C_{\mu}, k_{0}, k_{1}, i=1,2$ are positive constants. Let for any $\tau \in \mathbb{R}, \omega \in \Omega$, there is a time $T_{1}=T_{1}\left(B_{0}, \omega\right)$ satisfies

$$
\left.\hat{B}(\omega)=\sqcup_{t \geq \hat{T}} \chi\left(\tau, \tau-t, \theta_{-\tau} \omega ; \chi_{\tau-t}(\omega)\right)=\chi_{\tau-t}(\omega) \in \hat{B}\left(\tau, \theta_{-\tau} \omega\right)\right) \subseteq B_{0}(\omega), \forall t \geq \hat{T},
$$

for any $\omega \in \Omega$, where $\hat{T}=\hat{T}\left(B_{0}, \omega\right) \geq \tau$ is the pullback absorbing time in Lemma 2, then it holds $\hat{B}(\omega) \subseteq$ $B_{0}(\omega)$ that

$$
\left.\Phi\left(t, \tau, \theta_{-t} \omega ; \hat{B}\left(\tau, \theta_{-t} \omega\right)\right)=\chi\left(r, \tau-t, \theta_{-\tau} \omega ; \hat{B}\left(\tau, \theta_{-\tau} \omega\right)\right) \subseteq \hat{B}\left(\tau, \theta_{-\tau} \omega\right)\right) \subseteq B_{0}(\omega), \forall t \geq \check{T} .
$$

In order to obtain the regularity estimates, we decompose the solution $\chi(t, \tau, \omega)=$ $\left(u(t, \tau, \omega), v(t, \tau, \omega), \eta^{t}(t, \tau, s, \omega)\right)^{\top}$ of system (6) with initial data $\chi(\tau, \omega)=\left(u_{0}, v_{0}, \eta_{0}^{t}\right)^{\top}$ into two parts

$$
\left\{\begin{array}{l}
\chi(t, \tau, \omega)=\hat{\chi}(t, \tau, \omega)+\check{\chi}(t, \tau, \omega) \\
u=y+w \\
\eta^{t}=\hat{\eta}^{t}+\check{\eta}^{t}
\end{array}\right.
$$

Then, we can rewrite the Equation (6) into the following systems

$$
\left\{\begin{array}{l}
y_{t t}+\Delta^{2} y-k(0)\left(1+\int_{\Omega}|\nabla y|^{2} d x\right) \Delta y-\int_{0}^{\infty} \mu \Delta \hat{\eta} u(t-s) d s+a(x) g\left(y_{t}\right)+f_{1}(y)=\hat{q}(x, t), \\
\hat{\eta}_{t}=-\eta_{1 s}+y_{t} \\
y(\tau, x)=y_{0}(x), y_{t}(\tau, x)=y_{1}(x), x \in \Gamma, \tau \in \mathbb{R} \\
\hat{\eta}_{\tau}(x, \tau, s)=y_{0}(x)-y_{0}(x,-s), x \in \Gamma, \tau \in \mathbb{R}, s \in \mathbb{R}^{+}
\end{array}\right.
$$

Let $\hat{x}(t, \omega)=\left(\hat{y}, \check{y}, \hat{\eta}^{t}(t, s)\right)^{\top}, \hat{y}=y$ and $\check{y}=\hat{y}_{t}+\varepsilon \hat{y}$, which are equivalent with

$$
\left\{\begin{array}{l}
\hat{\chi}^{\prime}+H(\hat{\chi})=\hat{F}(\hat{x}, t, \omega), \\
\hat{x}(\tau, \omega)=\left(\hat{y}_{0}(x), \hat{y}_{1}(x)+\varepsilon \hat{y}_{0}(x), \hat{\eta}_{0}\right)^{\top}, \hat{x}=(\hat{y}, \check{y}, \hat{\eta})^{\top},
\end{array}\right.
$$

where

$$
\begin{gathered}
H(\hat{x})=\left(\begin{array}{c}
\varepsilon \hat{y}-\check{y} \\
-\varepsilon \check{y}+\varepsilon^{2} \hat{y}+A \hat{y}+\int_{0}^{\infty} \mu(s) A^{\frac{1}{2}} \hat{\eta}(s) d s \\
\varepsilon \hat{y}-\check{y}+\hat{\eta}_{s}
\end{array}\right), \\
0 \\
\hat{F}(\hat{x}, \omega, t)=\left(\begin{array}{c}
\left(1+k(0) \int_{\Omega}|\nabla \hat{y}|^{2} d x\right) A^{\frac{1}{2}} y_{1}-a(x) g\left(\hat{y}_{t}\right)-f_{1}(\hat{y})+\hat{q}(x, t) \\
0
\end{array}\right),
\end{gathered}
$$


and

$$
\left\{\begin{array}{l}
w_{t t}+\Delta^{2} w-k(0)\left(1+\int_{\Omega}|\nabla w|^{2} d x\right) \Delta w-\int_{0}^{\infty} \mu \Delta \breve{\eta} d s+a(x) g\left(w_{t}\right)+f(u)-f_{1}(y)=\breve{q}(x, t)+\kappa \sum_{j=1}^{m} h_{j} \dot{W}_{j}, \\
\breve{\eta}_{t}=-\breve{\eta}_{s}+w_{t} \\
w(\tau, x)=w_{0}(\tau, x), w_{t}(\tau, x)=w_{1}(\tau, x), x \in \Gamma, \tau \in \mathbb{R} \\
\check{\eta}_{\tau}(x, \tau, s)=w_{0}(x, \tau)-w_{0}(x, \tau-s), x \in \Gamma, \tau \in \mathbb{R}, s \in \mathbb{R}^{+}
\end{array}\right.
$$

Since $\check{\chi}=\left(\hat{w}, \check{w}, \breve{\eta}^{t}\right)^{\top},\left(\hat{w}, \check{w}=\hat{w}_{t}+\delta \hat{w}-\kappa z\left(\theta_{t} \omega\right), \check{\eta}^{t}\right)^{\top}$,

$$
\check{w}=\hat{w}_{t}+\delta \hat{w}-\kappa z\left(\theta_{t} \omega\right) .
$$

The above equations leads to

$$
\left\{\begin{array}{l}
\check{\chi}^{\prime}+H(\check{\chi})=\check{F}(\check{\chi}, t, \omega) \\
\check{\chi}(\tau, \omega)=\left(\hat{w}_{0}(x), \hat{w}_{1}(x)+\varepsilon \hat{w}_{0}(x)-\kappa z\left(\theta_{t} \omega\right), \check{\eta}_{0}\right)^{\top}, \check{\chi}=(\hat{w}, \check{w}, \check{\eta})^{\top}
\end{array}\right.
$$

in which

$$
H(\check{\chi})=\left(\begin{array}{c}
\varepsilon \hat{w}-\check{w} \\
-\varepsilon \check{w}+\varepsilon^{2} \hat{w}+A \hat{w}+\int_{0}^{\infty} \mu(s) A^{\frac{1}{2}} \check{\eta}(s) d s \\
\varepsilon \hat{w}-\check{w}+\check{\eta}_{s}
\end{array}\right)
$$

and

$$
\check{F}(\check{\chi}, \omega, t)=\left(\begin{array}{c}
\kappa z\left(\theta_{t} \omega\right) \\
\left(1+k(0) \int_{\Omega}|\nabla \hat{w}|^{2} d x\right) A^{\frac{1}{2}} \hat{w}-a(x) g\left(\hat{w}_{t}\right)-f(u)+f_{1}(\hat{y})+\hat{q}(x, t)+\kappa z\left(\theta_{t} \omega\right) \\
\kappa z\left(\theta_{t} \omega\right)
\end{array}\right) .
$$

Now we need to establish some priori estimates for the solutions of Equation (50) and Equation (53), which are the basis of our later analysis.

Lemma 3. Let (2)-(5),(7)-(8) and (45) hold. Let $\hat{B}(\tau, \omega) \subseteq B_{0}(\tau, \omega), \hat{B}=\{\hat{B}(\tau, \omega)\}_{\omega \in \Omega} \in D(E)$ and $\hat{\chi}_{0}(\omega) \in$ $\hat{B}(\tau, \omega)$. Then there exists $\hat{T}=\hat{T}(\hat{B}, \omega)>0$ and $M_{0}(\omega)$, such that the solution $\hat{\chi}\left(\hat{T}, \omega, \hat{\chi}_{\tau}(\omega)\right)$ of $(50)$ satisfies for P-a.e $\omega \in \Omega, \forall t \geq \hat{T}$

$$
\left\|\hat{\chi}\left(r, \tau-t, \omega, \hat{\chi}_{\tau-t}(\omega)\right)\right\|_{E}^{2} \leq\left\|\hat{\chi}_{\tau-t}\right\|^{2} e^{-2 \sigma t}+\int_{\tau}^{r} \hat{r}(\omega) d r \leq \hat{M}(\omega) .
$$

Proof. Taking inner product of (50) with $\hat{\chi}$ in $E$, we have

$$
\left(\hat{\chi}^{\prime}, \hat{\chi}\right)+(H(\hat{\chi}), \hat{\chi})=(\hat{F}(t, x, \hat{\chi}) \cdot \hat{\chi})
$$

Using Hölder, Young and Poincarè inequalities, we get

$$
(H(\hat{\chi}), \hat{\chi})=\varepsilon\|\Delta \hat{y}\|^{2}+\varepsilon^{2}(\hat{y}, \check{y})-\varepsilon\|\check{y}\|^{2}-\frac{m_{0} \varepsilon^{2}}{2}\|\nabla \hat{y}\|^{2}+\frac{\delta}{4}\|\nabla \hat{\eta}\|_{\mu}^{2} .
$$

Now, we estimate the terms on the right hand side of (55) one by one:

$$
\begin{aligned}
\left(\left(1+k(0)\|\nabla \hat{y}\|^{2}\right) \triangle \hat{y}, \check{y}\right) & =\left(\left(1+k(0)\|\nabla \hat{y}\|^{2}\right) \nabla \hat{y}, \nabla\left(\frac{d \hat{y}}{d t}+\varepsilon \hat{y}\right)\right) \\
& \leq\left(1+k(0)\|\nabla \hat{y}\|^{2}\right)\left(\frac{1}{2} \frac{d}{d t}\|\nabla \hat{y}\|^{2}+\frac{\varepsilon}{2}\|\nabla \hat{y}\|^{2}\right),
\end{aligned}
$$

and from (2), it is easy to show that

$$
\left(a(x) g\left(\hat{y}_{t}\right), \check{y}\right)=\left(\alpha_{0} g(\vartheta)(\check{y}-\varepsilon \hat{y}, \check{y})\right) \leq \alpha_{0} \alpha_{1}\|\check{y}\|^{2}-\alpha_{0} \alpha_{2} \varepsilon(\hat{y}, \check{y}),
$$


where $\vartheta$ is between 0 and $\check{y}-\varepsilon \hat{y}$.

$$
(\hat{q}(x, t), v)=\|\hat{q}(x, t)\|\|\check{y}\| \leq \frac{\|\hat{q}(x, t)\|^{2}}{\left(\alpha_{0} \alpha_{1}-\varepsilon\right)}+\frac{\alpha_{0} \alpha_{1}-\varepsilon}{4}\|\check{y}\|^{2},
$$

by second term for right hand side of (55) and (59), we can get

$$
\varepsilon\left(\varepsilon-\alpha_{2} \alpha_{0}\right)(\hat{y}, \check{y}) \geq-\frac{\alpha_{0} \alpha_{2} \varepsilon}{\lambda}\|\nabla \hat{y}\|\|\check{y}\| \geq \frac{\alpha_{0} \alpha_{1}-\varepsilon}{4}\|\check{y}\|^{2}-\frac{\alpha_{0}^{2} \alpha_{2}^{2} \varepsilon^{2}}{\left(\alpha_{0} \alpha_{1}-\varepsilon\right) \lambda^{2}}\|\nabla \hat{y}\|^{2},
$$

Further, from (45) $)_{\left(\mathbf{A}_{2}\right),\left(\mathbf{A}_{4}\right)}$, we infer

$$
\left.\left(f_{1}(\hat{y}), \check{y}\right)=\left(f_{1}(\hat{y}), \frac{d \hat{y}}{d t}+\varepsilon \hat{y}\right) \geq \frac{d}{d t} F_{1}(\hat{y})+\frac{\varepsilon}{k_{1}}\left(F_{1}(\hat{y})-c_{\mu}|\Gamma|\right)\right),
$$

Thus, applying together in (55) we conclude that

$$
\begin{aligned}
& \frac{1}{2} \frac{d}{d r}\left(\|\check{y}\|^{2}+\left(1+k(0)\|\nabla \hat{y}\|^{2}\right)\|\nabla \hat{y}\|^{2}+\|\nabla \hat{y}(r)\|^{2}+\|\nabla \hat{\eta}\|_{\mu}^{2}+\tilde{F}_{1}(\hat{y})\right) \\
& \quad+\frac{\varepsilon}{2}\left(\|\check{y}\|^{2}+\left(1+k(0)\|\nabla \hat{y}\|^{2}\right)\|\nabla \hat{y}\|^{2}\right)+\varepsilon\left(\frac{1}{\lambda}-\frac{m \varepsilon}{2}-\frac{\varepsilon \alpha_{0}^{2} \alpha_{2}^{2}}{\left(\alpha_{0} \alpha_{1}-\varepsilon\right) \lambda^{2}}\right)\|\nabla \hat{y}(r)\|^{2} \\
& \quad+\frac{\alpha_{0} \alpha_{1}-\varepsilon}{2}\|\check{y}\|^{2}+\frac{\delta}{4}\|\hat{\eta}(r)\|_{\mu, 1}^{2}+\frac{\varepsilon}{k_{1}} \hat{F}_{1}(y(r)) \\
& \leq \frac{\|q(x, t)\|^{2}}{\left(\alpha_{0} \alpha_{1}-\varepsilon\right)}+\frac{\varepsilon}{k_{1}} c_{\mu}|\Gamma|
\end{aligned}
$$

Since the inequalities above has nonnegative terms, we obtain $\varepsilon\left(\frac{1}{\lambda}-\frac{m \varepsilon}{2}-\frac{\varepsilon \alpha_{0}^{2} \alpha_{2}^{2}}{\left(\alpha_{0} \alpha_{1}-\varepsilon\right) \lambda^{2}}\right)>0, \frac{\alpha_{0} \alpha_{1}-\varepsilon}{2}>0$. We will choose $\sigma=\left(\frac{\varepsilon}{2}, \frac{\alpha_{0} \alpha_{1}-\varepsilon}{2}, 2 \varepsilon\left(\frac{1}{\lambda}-\frac{m \varepsilon}{2}-\frac{\varepsilon \alpha_{0}^{2} \alpha_{2}^{2}}{\left(\alpha_{0} \alpha_{1}-\varepsilon\right) \lambda^{2}}\right)\right)$ and $\hat{\sigma}=\min \left\{\sigma, \frac{\varepsilon}{k_{1}}, \frac{\delta}{4}\right\}$, which obviously implies that

$$
\frac{d}{d t}\|\hat{\chi}(r)\|_{E}^{2}+\hat{\sigma}\|\hat{\chi}(r)\|_{E}^{2} \leq \frac{\|\hat{q}(x, t)\|^{2}}{\left(\alpha_{0} \alpha_{1}-\varepsilon\right)}+\frac{\varepsilon}{k_{1}} c_{\mu}|\Gamma| .
$$

Note that $\hat{\chi}\left(r, \tau-t, \omega, \hat{\chi}_{\tau-t}(\omega)\right)=\chi\left(r, \tau-t, \omega, \chi_{\tau-t}(\omega)\right)-\left(0, z\left(\theta_{t} \omega\right), 0\right) \in B_{0}(\tau, \omega)$. By definition of $B_{0}(\tau, \omega)$, it follows that $\left\|\hat{\chi}\left(r, \tau-t, \omega, \hat{\chi}_{\tau-t}(\omega)\right)\right\|_{E}^{2} \leq \hat{r}(\omega)+\left|z\left(\theta_{r-\tau} \omega\right)\right|=\hat{M}(\omega)$. Now, by the Gronwall inequality to $[\tau-t, r]$, we arrive to (54); $\left\|\hat{\chi}\left(r, \tau-t, \omega, \hat{\chi}_{\tau-t}(\omega)\right)\right\|_{E}^{2} \leq \hat{M}(\omega)$. Hence, for every $\hat{y} \in H_{0}^{1}$,by $H_{0}^{1} \subset L^{\frac{2 n}{n-2}}$ and (62), we have

$$
0 \leq\left|\int_{\Omega} f_{1}(u)\right| d x \leq \mu_{1}\left(\left\|\left.\hat{y}\right|^{2}+\right\| \hat{y} \|^{\frac{2 n}{n-2}} \frac{2 n}{n-2}^{\frac{2 n}{n-2}}\right) \leq \hat{r}(\omega) \|\left.\nabla \hat{y}\right|^{2} \cdot \forall u \in \mathbb{R}, n \geq 3 .
$$

The proof is completed.

Lemma 4. Suppose (2)-(4) hold. Let $B_{1}(\omega) \subseteq B_{0}(\omega), \hat{B}=\{\hat{B}(\omega)\}_{\omega \in \Omega} \in \mathcal{D}(E)$ and $\check{\chi}_{\tau}(\omega) \in \hat{B}(\omega)$, then there exists $\breve{T}=\breve{T}(\hat{B}, \omega)>$,0 and a random radius $\check{M}(\omega)$, such that the solution $\check{\chi}\left(t, \tau, \omega, \check{\chi}_{\tau}(\omega)\right)$ of (53) satisfies for P-a.e $\omega \in \Omega, \forall t \geq \check{T}$

$$
\left\|A^{v} \check{\chi}\left(r, \tau-t, \theta_{-t} \omega, \check{\chi}_{\tau-t}\left(\theta_{-t} \omega\right)\right)\right\|_{E}^{2} \leq\left\|A^{v} \check{\chi}_{\tau-t}\left(\theta_{-t} \omega\right)\right\|^{2} e^{-2 \sigma(\tau-t)}+\check{r}(\omega) \leq \check{M}(\omega), \quad t \geq \tau .
$$

We denote

$$
v \in\left(0, \min \left\{\frac{1}{4}, \frac{n+2-(n-2) \gamma}{4}\right\}\right), \forall 1 \leq \gamma \leq \frac{n+2}{n-2}
$$

Proof. By (64), (23) and $\check{\chi}=\chi-\hat{\chi}$, there exists a random variable $r(\omega)>0$ such that

$$
\max \left\{\|\chi(0, \omega, \chi(0, \omega))\|_{E},\|\check{\chi}((0, \omega, \check{\chi}(0, \omega)))\|_{E}\right\} \leq r(\omega) .
$$


By the embedding relations, we have $\mathbf{V}_{v_{1}} \subset \mathbf{V}_{v_{2}}$, if $v_{1} \geq v_{2}$ and $\mathbf{V}_{v} \subset \mathbb{L}^{q}$, where $\frac{1}{q}=\frac{1}{2}-\frac{v}{n}, \frac{1}{\bar{q}}=\frac{1}{2}-\frac{1}{q}$ and $H_{0}^{v}=D\left(A^{\frac{v}{2}}\right)=\mathbf{V}_{v} \subset \mathbb{L}^{q} \subset \mathbb{L}^{\grave{q}} \subset \mathbf{V}_{-v}=D\left(A^{\frac{-v}{2}}\right)$. Multiplying (53) with $A^{v} \check{\chi}(r)$ and integrating over $\Gamma$, we can get

$$
\begin{aligned}
\left(H(\check{\chi}(r)), A^{v} \check{\chi}(r)\right) & =\left(\begin{array}{c}
\varepsilon u-v \\
-\varepsilon v+\varepsilon^{2} u+A u+\int_{0}^{\infty} \mu(s) A^{\frac{1}{2}} \eta(s) d s \\
\varepsilon u-v+\eta_{s}
\end{array}\right)\left(\begin{array}{c}
A^{v} u \\
A^{v} v \\
A^{v} \eta
\end{array}\right) \\
& =\varepsilon\left\|A^{\frac{v+1}{2}} \hat{w}\right\|^{2}+\varepsilon^{2}\left(\hat{w}, A^{v} \breve{w}\right)-\varepsilon\left\|A^{\frac{v}{2}} \check{w}\right\|^{2}-\frac{m_{0} \varepsilon^{2}}{2}\left\|A^{\frac{1+2 v}{4}} \hat{w}\right\|^{2}+\frac{\delta}{4}\left\|A^{\frac{1+2 v}{4}} \breve{\eta}\right\|_{\mu}^{2} .
\end{aligned}
$$

Now using Cauchy-Schwartz inequality and Young inequality one by one as

$$
\begin{aligned}
& \left(\mathbf{F}(t, x, \chi), A^{v} \chi\right)= \\
& \left(\begin{array}{c}
k h(x) z\left(\theta_{t} \omega\right) \\
\left(1+k(0)\left\|A^{\frac{1}{4}} \hat{w}\right\|^{2}\right) A^{\frac{1}{2}} \hat{w}-a(x) g\left(\hat{w}_{t}\right)+f_{1}(\hat{y})-f(u)+\check{q}(x, t)+\kappa h(x) z\left(\theta_{t} \omega\right) \\
k h(x) z\left(\theta_{t} \omega\right)
\end{array}\right)\left(\begin{array}{c}
A^{v} \hat{w} \\
A^{v} \breve{w} \\
A^{v} \check{\eta}
\end{array}\right) .
\end{aligned}
$$

From $(3)_{\left(\mathbf{A}_{2}\right),\left(\mathbf{A}_{4}\right)}$, one has that

$$
\begin{aligned}
& \left.-\left(1+k(0)\left\|A^{\frac{1}{4}} \hat{w}\right\|^{2}\right) A^{\frac{1}{2}} \hat{w}, A^{v} \check{w}\right)=\left(\left(1+k(0)\left\|A^{\frac{1}{4}} \hat{w}\right\|^{2}\right) A^{\frac{v}{2}} \hat{w}, A^{\frac{v}{2}}\left(\frac{d \hat{w}}{d t}+\varepsilon \hat{w}-a h(x) z\left(\theta_{t} \omega\right)\right)\right. \\
& \left.\leq-\left(1+k(0)\left\|A^{\frac{1}{4}} \hat{w}\right\|^{2}\right)\left(\frac{1}{2} \frac{d}{d t}\left\|A^{\frac{v}{2}} \hat{w}\right\|^{2}+\frac{\varepsilon}{2}\left\|A^{\frac{v}{2}} \hat{w}\right\|^{2}\right)+\frac{\kappa^{2}}{2 \varepsilon} \| A^{\frac{v}{2}} h(x)\right) \|^{2}\left|z\left(\theta_{t} \omega\right)\right|^{2} .
\end{aligned}
$$

Therefore from (2) and (4), it is straightforward to show that

$$
\begin{aligned}
& \left(a(x) g\left(\hat{w}_{t}\right), A^{v} \breve{w}\right)=-\left(\alpha_{0} g(\vartheta) A^{\frac{v}{2}}\left(\check{w}-\varepsilon \hat{w}+\kappa h(x) z\left(\theta_{t} \omega\right)-g(0)\right), A^{\frac{v}{2}} \check{w}\right) \\
& \leq-\alpha_{0} \alpha_{1}\left\|A^{\frac{v}{2}} \check{w}\right\|^{2}+\alpha_{0} \alpha_{2} \varepsilon\left(A^{\frac{v}{2}} \hat{w}, A^{\frac{v}{2}} \check{w}\right)-\alpha_{0} g^{\prime}(\vartheta) \kappa\left(h(x) z\left(\theta_{t} \omega, A^{\frac{v}{2}} \check{w}\right),\right.
\end{aligned}
$$

where $\vartheta$ is between 0 and $\check{w}-\varepsilon \hat{w}+\kappa h(x) z\left(\theta_{t} \omega\right)$.

$$
\begin{aligned}
& \left(\breve{q}(x, t), A^{v} \breve{w}\right)=\left\|A^{\frac{v}{2}} \breve{q}(x, t)\right\|\left\|A^{\frac{v}{2}} \check{w}\right\| \leq \frac{\left\|A^{\frac{v}{2}} \breve{q}(x, t)\right\|^{2}}{2\left(\alpha_{0} \alpha_{1}-\varepsilon\right)}+\frac{\alpha_{0} \alpha_{1}-\varepsilon}{2}\left\|A^{\frac{v}{2}} \check{w}\right\|^{2}, \\
& \left(\left(k \varepsilon h(x) z\left(\theta_{t} \omega\right), A^{v} \hat{w}\right)\right) \leq \frac{\varepsilon}{4}\left\|A^{\frac{2 v+1}{2}} \hat{w}\right\|^{2}+\varepsilon k^{2}\left\|A^{\frac{2 v+1}{2}} h(x)\right\|^{2}\left|z\left(\theta_{t} \omega\right)\right|^{2}, \\
& \left(k \varepsilon h(x) z\left(\theta_{t} \omega\right), A^{v} \breve{\eta}\right)_{\mu} \leq \frac{2 m_{0} k^{2}}{\delta}\left\|A^{\frac{2 v+1}{4}} h(x)\right\|^{2}\left|z\left(\theta_{t} \omega\right)\right|^{2}+\frac{\delta}{8}\left\|A^{\frac{1+2 v}{4}} \check{\eta}\right\|_{\mu}^{2}, \\
& \left.-\left(\alpha_{0} g^{\prime}(\vartheta)-2 \varepsilon\right) \kappa h(x) z\left(\theta_{t} \omega\right), A^{v} \breve{w}\right) \leq \frac{2\left(\alpha_{0} \alpha_{2} \kappa\right)^{2}}{\alpha_{0} \alpha_{1}-\varepsilon}\left\|A^{\frac{v}{2}} h(x)\right\|^{2}\left|z\left(\theta_{t} \omega\right)\right|^{2}+\frac{\alpha_{0} \alpha_{1}-\varepsilon}{4}\left\|A^{\frac{v}{2}} \breve{w}\right\|^{2} .
\end{aligned}
$$

Through 2d term for right hand side of (26) and (29), we will get

$$
\varepsilon\left(\varepsilon-\alpha_{2} \alpha_{0}\right)\left(\hat{w}, A^{v} \breve{w}\right) \geq-\alpha_{0} \alpha_{2} \varepsilon\left\|A^{\frac{v}{2}} \hat{w}\right\|\left\|A^{\frac{v}{2}} \breve{w}\right\| \geq \frac{\alpha_{0} \alpha_{1}-\varepsilon}{4}\left\|A^{\frac{v}{2}} \check{w}\right\|^{2}-\frac{\left(\alpha_{0} \alpha_{2} \varepsilon\right)^{2}}{\left(\alpha_{0} \alpha_{1}-\varepsilon\right) \lambda^{2}}\left\|A^{\frac{1+2 v}{4}} \hat{w}\right\|^{2} .
$$

For the nonlinearity, with the aid of (4), Hölder inequality and the Sobolev embedding theorem, we estimate that

$$
\begin{aligned}
& \left(f(u)-f_{1}(\hat{y}), A^{v} \breve{w}\right)=\left(f(u)-f_{1}(\hat{y}), A^{v}\left(\hat{w}_{t}+\varepsilon \hat{w}-\kappa h(x) z\left(\theta_{t} \omega\right)\right)\right) \\
& \leq \frac{d}{d t} \int_{\Gamma}\left(f(u)-f_{1}(\hat{y})\right) A^{v} \hat{w} d x+\varepsilon \int_{\Gamma}\left(f(u)-f_{1}(\hat{w})\right) A^{v} \hat{w} d x \\
& -\int_{\Gamma}\left(f^{\prime}(u) u_{t}-f_{1}^{\prime}(\hat{w}) \hat{w}_{t}\right) A^{v} \hat{w} d x-\kappa \int_{\Gamma}\left(f(u)-f_{1}(\hat{w})\right)\left|A^{v} h(x)\right|\left|z\left(\theta_{t} \omega\right)\right| d x
\end{aligned}
$$

Infer to $\mathbf{A}_{4}$, (45)-(46), use Cauchy-Schwartz, Young's inequality and embedding theorem $\mathbf{V}_{1+v} \subset L^{\frac{2 n}{n-2(1-v)}}$, $\mathbf{V}_{1-v} \subset L^{\frac{2 n}{n+2(1-v)}}$ and $\mathbf{V}_{1} \hookrightarrow L^{\frac{2 n}{n-2}}$, we gain 


$$
\begin{aligned}
& \int_{\Gamma}\left(\left(f(u)-f_{1}(\hat{w})\right) A^{v}\left(\kappa\left|h(x) \| z\left(\theta_{t} \omega\right)\right|\right) d x \leq \int_{\Gamma}\left(\left(f_{1}(u)+f_{2}(u)-f_{1}(\hat{w})\right) A^{v} \kappa\left(\left|h(x) \| z\left(\theta_{t} \omega\right)\right|\right) d x\right.\right. \\
& \leq \mu_{1} \mathcal{\kappa}\left(\int_{\Gamma}\left(1+|\hat{y}|^{\frac{4}{n-2}}+|\hat{w}|^{\frac{4}{n-2}}\right)^{\frac{2 n}{4}} d x\right)^{\frac{4}{2 n}}\left(\int_{\Gamma}|\hat{w}|^{\frac{2 n}{n-2(1+v)}} d x\right)^{\frac{n-2(1+v)}{2 n}}\left\|A^{v} h(x)\right\|\left|z\left(\theta_{t} \omega\right)\right| \\
& +\mu_{2} \kappa\left(\int_{\Gamma}\left(1+|\hat{u}|^{\gamma}\right)^{\frac{2 n}{n+2(1-v)}} d x\right)^{\frac{n+2(1-v)}{2 n}}\left\|A^{v} h(x)\right\|\left|z\left(\theta_{t} \omega\right)\right| \\
& \left.\leq \mu_{3}\left(1+\|u\|_{L^{\frac{2 n}{n-2}}}^{\frac{2}{n-2}}+\|u\|^{\frac{4}{n-2}}\right) \| \hat{\frac{2 n}{n-2}}\right)\|\|_{L^{\frac{2 n}{n-2(1+v)}}}\left\|A^{\frac{v}{2}} h(x)\right\|_{L^{1+2 n}}\left|z\left(\theta_{t} \omega\right)\right| \\
& +\mu_{4}\left(1+\|u\|_{L_{\frac{2 n}{n+2(1-v)}}^{\gamma}}\right)\left\|A^{\frac{v}{2}} h(x)\right\|_{L^{\frac{2 n}{1+2 v}}}\left|z\left(\theta_{t} \omega\right)\right| \\
& \leq \mu_{5}\left(r_{1}^{2}(\omega)+\left\|A^{\frac{1+v}{2}} \hat{w}\right\|^{2}\right)+\mu_{6}\left\|A^{v} h(x)\right\|^{2}\left|z\left(\theta_{t} \omega\right)\right|^{2} .
\end{aligned}
$$

and therefore

$$
\int_{\Gamma}\left(f^{\prime}(u) u_{t}-f_{1}^{\prime}(\hat{w}) \hat{w}_{t}\right) A^{v} \hat{w} d x=\int_{\Gamma}\left(\left(f_{1}^{\prime}(u)-f_{1}^{\prime}(\hat{w})\right) u_{t}+f_{1}^{\prime}(\hat{w}) \hat{w}_{t}+f_{2}^{\prime}(u) u_{t}\right) A^{v} \hat{w} d x .
$$

Estimate the above inequality, we get

$$
\begin{aligned}
& \int_{\Gamma}\left(f_{1}^{\prime}(u)-f_{1}^{\prime}(\hat{y})\right) u_{t} A^{v} \breve{w} d x \leq \mu_{7} \int_{\Gamma}\left(1+|\hat{y}|^{\frac{6-n}{n-2}}+|\hat{w}|^{\frac{6-n}{n-2}}\right)\left|\hat{w} \| A^{v} \hat{w}\right|\left|u_{t}\right| d x \\
& \leq \mu_{8}\left(\int_{\Gamma}\left(1+|\hat{y}|^{\frac{6-n}{n-2}}+|\hat{w}|^{\frac{6-n}{n-2}}\right)^{\frac{2 n}{6-n}} d x\right)^{\frac{6-n}{2 n}}\left(\int_{\Gamma}\left|u_{t}\right|^{2} d x\right)^{\frac{1}{2}}\left(\int_{\Gamma}|\hat{w}|^{\frac{2 n}{n-2(1+v)}} d x\right)^{\frac{n-2(1+v)}{2 n}}\left(\int_{\Gamma}\left|A^{v} \hat{w}\right|^{\frac{2 n}{n-2(1-v)}} d x\right)^{\frac{n-2(1-v)}{2 n}} \\
& \leq \mu_{9}\left(1+\|\hat{y}\|_{L^{\frac{2 n}{n-2}}}^{\frac{6-n}{n-2}}+\|\hat{w}\|_{L^{\frac{2 n}{n-2}}}^{\frac{6-n}{n-2}}\right)\left\|u_{t}\right\|_{L^{2}}\|\hat{w}\|_{L^{\frac{2 n}{n-2(1+v)}}\left\|A^{v} \hat{w}\right\|_{L^{\frac{2 n}{n-2(1-v)}}}} \\
& \leq \mu_{10}\left(\|\hat{w}\|_{L^{\frac{2 n}{n-2(1+v)}}}\left\|A^{v} \hat{w}\right\|_{L^{\frac{2 n}{n-2(1-v)}}}\right)+\mu_{11}\left(\|\hat{y}\|_{L^{\frac{2 n}{n-2}}}^{\frac{2^{n}}{n-2}}+\|\hat{w}\|_{L^{\frac{2-n}{n-2}}}^{\frac{2 n}{n-2}}\right)\|\hat{w}\|_{L^{\frac{2 n}{n-2(1+v)}}}\left\|A^{v} \hat{w}\right\|_{L^{\frac{2 n}{n-2(1-v)}}} \\
& \leq \mu_{12} r_{2}(\omega)\left(\left\|A^{\frac{v}{2}} \hat{w}\right\|^{2}+\left\|A^{\frac{1+v}{2}} \hat{w}\right\|^{2}\right) .
\end{aligned}
$$

Similarly, by (45) $\mathbf{A}_{2}$ and (65), we get

$$
\begin{aligned}
& \int_{\Gamma} f_{1}^{\prime}(\hat{y}) \hat{w}_{t} A^{v} \hat{w} d x \leq \mu_{13}\left(\int_{\Gamma}\left(1+|\hat{y}|^{\frac{4}{n-2}}\right)^{\frac{2 n}{4}} d x\right)^{\frac{4}{2 n}} \\
& \times\left(\int_{\Gamma}\left|\hat{w}_{t}\right|^{\frac{2 n}{n-2(1+v)}} d x\right)^{\frac{n-2(1+v)}{2 n}} \times\left(\int_{\Gamma}\left|A^{v} \hat{w}\right|^{\frac{2 n}{n-2(1-v)}} d x\right)^{\frac{n-2(1-v)}{2 n}} \\
& \leq \mu_{14}\left(1+\|\hat{y}\|_{L^{\frac{2 n}{n-2}}}^{\frac{4}{n-2}}\right)\left\|\hat{w}_{t}\right\|_{L^{\frac{2 n}{n-2(1+v)}}}\left\|A^{v} \hat{w}\right\|_{L \frac{2 n}{n-2(1-v)}} \\
& \leq \mu_{15} r_{3}(\omega)\left(\left\|A^{\frac{v}{2}} \hat{w}\right\|+|\varepsilon|\right)\left\|A^{\frac{1+v}{2}} \hat{w}\right\|_{L_{\frac{2 n}{n-2(1-v)}}} \\
& \leq \mu_{16} r_{3}(\omega)\left(\left\|A^{\frac{v}{2}} \hat{w}\right\|^{2}+|\varepsilon|^{2}\right)+\frac{\varepsilon}{16}\left\|A^{\frac{1+v}{2}} \hat{w}\right\|_{L \frac{6}{1+2 v}}^{2}
\end{aligned}
$$

Furthermore, by (45) $\mathbf{A}_{3}$ and (65), note that $v \leq \frac{n+2-(n-2) \gamma}{4}$

$$
\begin{aligned}
& \int_{\Gamma} f_{2}^{\prime}(u) u_{t} A^{v} \hat{w} d x \leq \mu_{17} \int_{\Gamma}\left(\left(1+|u|^{\gamma}\right)\left|u_{t} \| A^{v} \hat{w}\right|\right) d x \\
& \leq \mu_{18}\left(\int_{\Gamma}\left(1+\|u\|^{\gamma}\right)^{\frac{2 n}{n+2(1-v)}} d x\right)^{\frac{n+2(1-v)}{2 n}}\left(\int_{\Gamma}\left|u_{t}\right|^{2} d x\right)^{\frac{1}{2}}\left(\int_{\Gamma}\left|A^{v} \hat{w}\right|^{\frac{2 n}{n-2(1-v)}} d x\right)^{\frac{n-2(1-v)}{2 n}} \\
& \leq \mu_{19}\left(1+\|u\|_{L_{\frac{2 n}{n+2(1-v)}}^{\gamma}}\right)\left\|u_{t}\right\|_{L^{2}}\left\|A^{\frac{v}{2}} \hat{w}\right\|_{L_{\frac{2 n}{n-2(1-v)}}} \\
& \leq \mu_{20} r_{4}^{2}(\omega)+\frac{\varepsilon}{8}\left\|A^{\frac{1+v}{2}} \hat{w}\right\|^{2} .
\end{aligned}
$$


Including above inequalities together (66)-(78), we achieve

$$
\begin{aligned}
& \frac{1}{2} \frac{d}{d t}\left[\left\|A^{\frac{v}{2}} \check{\chi}\right\|_{E}^{2}+2\left(f(u)-f_{1}(\hat{w})\right)\right]+\frac{\varepsilon}{4}\left\|A^{\frac{v}{2}} \check{\chi}\right\|_{E}^{2}+\frac{\varepsilon}{2}\left(f(u)-f_{1}(\hat{w})\right) \\
& \leq|\kappa|\left|z\left(\theta_{t} \omega\right)\right|\left\|A^{\frac{v}{2}} \check{\chi}\right\|_{E}^{2}+\check{\mu}_{C}\left[1+r_{1}^{2}(\omega)+r_{2}^{2}(\omega)+r_{3}^{2}(\omega)+r_{4}^{2}(\omega)\right. \\
& \left.\quad+\left|z\left(\theta_{t} \omega\right)\right|^{2}+\left\|A^{v} h(x)\right\|^{2}\left|z\left(\theta_{t} \omega\right)\right|^{2}+\left\|A^{\frac{v}{2}} \check{q}(x)\right\|^{2}\right] .
\end{aligned}
$$

By Gronwall's inequality in (79) on $[0, r]$ and changing $\omega$ to $\theta_{-t} \omega$, we deduce that

$$
\begin{aligned}
& \left\|A^{\frac{\nu}{2}} \bar{\varphi}\left(t, \theta_{-t} \omega ; \varphi_{0}\right)\right\|_{E}^{2} \leq\left(\left\|A^{\frac{\nu}{2}} \varphi\left(r, \theta_{-t} \omega ; \varphi_{0}\right)\right\|_{E}^{2}+2\left(f\left(u\left(r, \theta_{-t} \omega ; \chi_{0}\right)\right)-f_{1}\left(\hat{w}\left(r, \theta_{-t} \omega ; \chi_{0}\right)\right)\right)\right. \\
& \leq\left(\left\|A^{\frac{v}{2}} \check{\chi}\right\|_{E}^{2}+\left(f(u)-f_{1}(\hat{w})\right)\right) \exp ^{2 \int_{r}^{0}\left(\sigma-|\kappa|\left|z\left(\theta_{s} \omega\right)\right|\right)(s, \omega) d s}+\int_{0}^{r} \varrho_{1}\left(\theta_{s} \omega\right) \exp ^{2 \int_{r}^{s}\left(\sigma-|\kappa|\left|z\left(\theta_{\zeta} \omega\right)\right|\right)(\varsigma, \omega) d s} d s .
\end{aligned}
$$

We can choose $\varrho_{1}\left(\theta_{t} \omega\right)$ and $\check{\mu}_{C}$ depends to $\left[\varepsilon, \delta, \kappa, \alpha_{0}, \alpha_{1}, \alpha_{2}, m_{0}, \mu_{i}\right]$ are positive constants, such that

$$
\begin{aligned}
& \varrho_{1}\left(\theta_{t} \omega\right)=\check{\mu}_{C}\left[1+r_{1}^{2}(\omega)+r_{2}^{2}(\omega)+r_{3}^{2}(\omega)+r_{4}^{2}(\omega)\right. \\
& \left.\quad+\left\|A^{\frac{v}{2}} h(x)\right\|^{2}\left|z\left(\theta_{t} \omega\right)\right|^{2}+\left\|A^{\frac{v}{2}} h(x)\right\|^{2}\left|z\left(\theta_{t} \omega\right)\right|^{2}+\left\|A^{\frac{v}{2}} \breve{q}(x)\right\|^{2}\right] .
\end{aligned}
$$

Note that

$$
\begin{aligned}
& \int_{\Gamma}\left(\left(f(u)-f_{1}(\hat{w})\right) A^{\frac{v}{2}} \hat{w} d x \leq \int_{\Gamma}\left(\left(f_{1}(u)+f_{2}(u)-f_{1}(\hat{w})\right) A^{\frac{v}{2}} \hat{w} d x\right.\right. \\
& \leq \mu_{21}\left(\int_{\Gamma}\left(1+|\hat{y}|^{\frac{4}{n-2}}+|\hat{w}|^{\frac{4}{n-2}}\right)|\hat{w}|\left|A^{\frac{v}{2}} \hat{w}\right| d x\right)+\mu_{22}\left(\int_{\Gamma}\left(1+|\hat{u}|^{\gamma}\right)\left|A^{\frac{v}{2}} \hat{w}\right| d x\right) .
\end{aligned}
$$

Thus, by the Sobolev embedding

$$
\begin{aligned}
& \left(\int_{\Gamma}\left(1+|\hat{y}|^{\frac{4}{n-2}}+|\hat{w}|^{\frac{4}{n-2}}\right)\right)|\hat{w}|+\left(\int_{\Gamma}\left(1+|\hat{u}|^{\gamma}\right) d x\right)\left|A^{\frac{v}{2}} \hat{w}\right| d x \\
& \leq \mu_{23}\left(\int_{\Gamma}\left(1+|\hat{y}|^{\frac{4}{n-2}}+|\hat{w}|^{\frac{4}{n-2}}\right)^{\frac{2 n}{4}} d x\right)^{\frac{4}{2 n}}\left(\int_{\Gamma}|\hat{w}|^{\frac{2 n}{n-2(1+v)}} d x\right)^{\frac{n-2(1+v)}{2 n}}\left(\int_{\Gamma}\left|A^{v} \hat{w}\right|^{\frac{2 n}{n-2(1-v)}} d x\right)^{\frac{n-2(1-v)}{2 n}} \\
& +\mu_{24}\left(\int_{\Gamma}\left(1+|\hat{u}|^{\gamma}\right)^{\frac{2 n}{n+2(1-v)}} d x\right)^{\frac{n+2(1-v)}{2 n}} \times\left(\int_{\Gamma}\left|A^{v} \hat{w}\right|^{\frac{2 n}{n-2(1-v)}} d x\right)^{\frac{n-2(1-v)}{2 n}} \\
& \leq \mu_{25} r_{5}^{2}(\omega)+\mu_{25}\left\|A^{\frac{1+v}{2}} \hat{w}\right\|^{2}
\end{aligned}
$$

where the constants $\mu_{i}, i=1,2, \ldots . ., 25$, comes from the embedding $D\left(A^{\frac{1+v}{2}}\right) \hookrightarrow L^{\frac{2 n}{n-2(1-v)}}, D\left(A^{\frac{1-v}{2}}\right) \hookrightarrow L^{\frac{2 n}{n-2(1+v)}}$ and $\mathbf{V}_{1}=H_{0}^{1} \hookrightarrow L^{\frac{2 n}{n-2}}$.

Note that, $\left|z\left(\theta_{\varsigma} \omega\right)\right|$ is tempered, and hence applying the inequalities (81) and (82) in (80), the integrand of the second term on the righthand side of (80) is convergent to zero exponentially as $r \hookrightarrow-\infty$. Then, we can shows that the following result

$$
\left\|A^{\frac{v}{2}} \check{\chi}\left(t, \theta_{-t} \omega ; \chi_{0}\right)\right\|_{E}^{2} \leq \check{M}_{2}^{2}(\omega)
$$

The proof is complete.

Now we obtain our main result about the existence of a random attractor for random dynamical system $\Phi$ as following Lemma. It follows from Lemma 2, that $\Phi$ has a closed random absorbing set in $\mathcal{D}$, then apply Lemmas in Section 4, we prove the existence of a random attractor by using tail estimates and the decompose technique of solutions. which along with the $\mathcal{D}$-pullback asymptotic compactness. 
Lemma 5. (see[2,3,15]) Let $\mathbf{X}_{\mathbf{0}}, \mathbf{X}, \mathbf{X}_{\mathbf{1}}$ be three Banach spaces such that $\mathbf{X}_{\mathbf{0}} \hookrightarrow \mathbf{X} \hookrightarrow \mathbf{X}_{\mathbf{1}}$ is projection operator $\mathbf{X}_{\mathbf{0}} \hookrightarrow \mathbf{X}$ is compact. setting $Y=\chi(t, \hat{B}(\tau, \omega)) \subset L_{\mu}^{2}\left(\mathbb{R}^{+}, \mathbf{X}\right)$ is a random bounded absorbing set from Lemma $4, \psi(t)$ is the solution operators of (53) and by Lemma 4, there is a positive random radius $M_{v}(\omega)$ dependent on $t$, such that

$$
\left.\begin{array}{l}
\text { 1). } \quad \text { Y is bounded in } L_{\mu}^{2}\left(\mathbb{R}^{+}, \mathbf{X}_{\mathbf{0}}\right) \bigcap H_{\mu}^{1}\left(\mathbb{R}^{+}, \mathbf{X}_{\mathbf{1}}\right), \\
\text { 2). } \sup _{\eta \in Y, s \in \mathbb{R}^{+}}\|\nabla \eta(s)\|_{\mathbf{X}}^{2} \leq M_{\nu}(\omega) .
\end{array}\right\}
$$

Then $Y$ is relatively compact in $L_{\mu}^{2}\left(\mathbb{R}^{+}, \mathbf{X}\right)$. Further, for every $\tau \in \mathbb{R}, \omega \in \Omega, t \geq 0$, so that

$$
\begin{gathered}
\check{\eta}\left(t, \tau, \theta_{-t} \omega, \chi_{0}\left(\theta_{-t} \omega\right), s\right)=\left\{\begin{array}{l}
\hat{w}\left(t, \tau, \theta_{-t} \omega, \chi_{0}\left(\theta_{-t} \omega\right)\right)-\hat{w}\left(t-s, \tau, \theta_{-t+s} \omega, \chi_{0}\left(\theta_{-t+s} \omega\right)\right), s \leq t, \\
\hat{w}\left(t, \tau, \theta_{-t} \omega, \chi_{0}\left(\theta_{-t} \omega\right)\right), t \leq s
\end{array}\right. \\
\check{\eta}_{s}\left(t, \tau, \theta_{-t} \omega, \chi_{0}\left(\theta_{-t} \omega\right)\right)=\left\{\begin{array}{l}
\hat{w}_{t}\left(t-s, \tau, \theta_{-t+s} \omega, \chi_{0}\left(\theta_{-t+s} \omega\right)\right), 0 \leq s \leq t, \\
0, t \leq s .
\end{array}\right.
\end{gathered}
$$

Denote by $\check{B}$ the closed ball of $L_{\mu}^{2}\left(\mathbb{R}^{+}, \mathbf{X}_{\mathbf{0}}\right) \cap H_{\mu}^{1}\left(\mathbb{R}^{+}, \mathbf{X}_{\mathbf{1}}\right)$ of random variable radius $M_{0}(\omega)$, since we apply on a finite domain. $\breve{B}$ is compact subset of $\mathbf{X}_{\mathbf{0}} \times \mathbf{X}_{\mathbf{1}}$. Let a set $\breve{B}(\tau, \omega)$

$$
\check{B}(\tau, \omega)=\overline{\bigcup_{\hat{\chi}_{\tau-t}\left(\theta_{-\tau} \omega\right) \in \check{B}\left(\theta_{-t} \omega\right)} \bigcup_{t \geq 0} \check{\eta}\left(\tau, \tau-t, \theta_{-\tau} \omega, \chi_{\tau-t}\left(\theta_{-\tau} \omega\right), s\right)} s \in \mathbb{R}^{+} \tau \in \mathbb{R}, \omega \in \Omega,
$$

where $v$ is as in (65). Thus, employing (3), Lemma 2, Lemma 4 and (84), we get that

$$
\sup _{\eta \in \breve{B}, s \in \mathbb{R}^{+}}\|\nabla \eta(s)\|_{\mu}^{2}=\sup _{t \geq 0} \sup _{\chi_{\tau-t}\left(\theta_{-\tau} \omega\right) \in B\left(\theta_{-t} \omega\right), s \in \mathbb{R}^{+}}\left\|\nabla \breve{\eta}\left(\tau, \tau-t,\left(\theta_{-\tau} \omega\right), \chi_{\tau-t}\left(\theta_{-\tau} \omega\right), s\right)\right\|^{2} \leq M_{0}(\omega),
$$

implying that

$$
\|\nabla \eta(s)\|_{\mu}^{2}=\int_{\tau}^{+\infty} \mu(s)\|\nabla \eta(s)\|^{2} d s \leq M_{0}(\omega) \int_{\tau}^{+\infty} e^{\sigma s} d s \leq \frac{M_{0}(\omega)}{\sigma} .
$$

We obtain our main result about the existence of a random attractor for random dynamical system $\Phi$ as following Theorem.

Theorem 3. Suppose (2)-(4) hold. Then the continuous cocycle $\Phi$ associated with Problem (16), has a unique D-pullback random attractor $\mathcal{A}(\tau, \omega) \in \mathcal{D}$ in $\Gamma$.

Proof. For any $(\tau, \omega) \in(\mathbb{R} \times \Omega)$. Let $\hat{\chi}_{\tau-t}\left(\theta_{-\tau} \omega\right) \in \hat{B}\left(\tau, \theta_{-t} \omega\right), \check{B} \subset \hat{B}\left(\theta_{-t} \omega\right)$ is compact in $\mathcal{D}(E)$. It follows that $\breve{B}$ be the closed ball of $\mathbf{V}_{2 v+1} \times \mathbf{V}_{2 v} \hookrightarrow E$ is compact of radius $\hat{M}(\omega) \in \mathcal{D}(E)$, where $v$ satisfy (65). Therefore, $\Lambda(\tau, \omega)$ is compact in $E$ for any bounded non-random set $B$ of $E$. By Lemma 3 and $\chi_{\tau-t}\left(\theta_{-\tau} \omega\right) \in$ $\breve{B}\left(\tau, \theta_{-t} \omega\right)$, we have $\chi_{\tau-t}=\check{\chi}_{\tau-t}-\hat{\chi}_{\tau-t} \in \Lambda(\tau, \omega)$, where $\chi_{\tau-t}$ is given by (50). Then, there exists a random set $\hat{M}(\omega) \in \check{B} \subseteq B(\tau, \omega) \in \mathcal{D}(E)$, as follows

$$
d_{H}\left(\Phi\left(t, \tau, \theta_{-t} \omega, B\left(\tau, \theta_{-t} \omega\right)\right), \Lambda(\tau, \omega)\right) \leq \hat{M}(\omega) e^{-\sigma t} \rightarrow 0, \text { as } t \rightarrow+\infty .
$$

From Lemma 3, there exists $\hat{T}=\hat{T}(\tau, \omega, B) \geq 0$, then we dedicate the following attraction property

$$
\hat{\chi}\left(\tau, \tau-t, \theta_{-\tau} \omega, B\left(\tau, \theta_{-t} \omega\right)\right) \subseteq B_{0}(\tau, \omega), \forall t \geq \hat{T} .
$$

Let $t \geq \hat{T}$ and $\breve{T}=t-\hat{T} \geq T\left(\tau, \omega, B_{0}\right) \geq 0$ using cocycle property (iii) of $\Phi$, we show that

$$
\begin{aligned}
& \hat{\chi}\left(\tau, \tau-t, \theta_{-\tau} \omega, B\left(\tau-t, \theta_{-\tau} \omega\right)\right) \\
& =\hat{\chi}\left(t, \tau-\check{T}-\hat{T}, \theta_{-t} \omega, B\left(\tau-\check{T}-\hat{T}, \theta_{-\tau} \omega\right)\right) \\
& =\hat{\chi}\left(\tau, \tau-\check{T},\left(\theta_{-\tau} \omega\right), \chi\left(\tau-\check{T}, \tau-\hat{T}-\check{T}, \theta_{-\tau} \omega, B\left(\tau-\check{T}-\hat{T}, \theta_{-\tau} \omega\right)\right)\right. \\
& \subseteq \hat{\chi}\left(\tau, \tau-\breve{T}, \theta_{-\tau} \omega, B_{0}\left(\theta_{-\check{T}} \omega\right)\right) \subseteq \hat{B}\left(\tau, \theta_{-\tau} \omega\right) .
\end{aligned}
$$


Take any $\hat{\chi}\left(\tau, \tau-t,\left(\theta_{-\tau} \omega\right), \chi_{\tau-t}\left(\theta_{-\tau} \omega\right)\right) \in \hat{\chi}\left(\tau, \tau-t, \theta_{-\tau} \omega, B\left(\tau-t, \theta_{-t} \omega\right)\right)$, for $t \geq \hat{T}+T\left(\tau, \omega, B_{0}\right)$, where $\hat{\chi}_{\tau-t}\left(\theta_{-\tau} \omega\right) \in B\left(\tau-t, \theta_{-t} \omega\right)$. It follow to Lemmas 2,3 and (90), such that

$$
\begin{aligned}
& \inf _{\eta \in \Lambda(\tau, \omega)}\left\|\chi\left(\tau, \tau-t, \theta_{-\tau} \omega, \chi_{\tau-t}\left(\theta_{-\tau} \omega\right)\right)-\eta\left(\tau, \tau-t, \theta_{-\tau} \omega, \chi_{\tau-t}\left(\theta_{-\tau} \omega\right)\right)\right\|_{E}^{2} \\
& \leq\left\|y\left(\tau, \tau-t, \theta_{-\tau} \omega, y_{\tau-t}\left(\theta_{-\tau} \omega\right)\right)\right\|_{E}^{2} \leq \hat{M}^{2}(\omega) e^{-\sigma_{3} t}, \forall t>\hat{T}+T\left(\tau, \omega, B_{0}\right) .
\end{aligned}
$$

Thus from the relation (19) between $\hat{\boldsymbol{\Phi}}, \breve{\boldsymbol{\Phi}}$, one could easily obtain that for any nonrandom bounded

$$
d_{H}\left(\overline{\boldsymbol{\Phi}}\left(t, \tau, \theta_{-t} \omega, B\left(\tau, \theta_{-t} \omega\right)\right), \Lambda(\tau, \omega)\right) \leq \hat{M}(\omega) e^{-\sigma_{3} t} \rightarrow 0 \text { as } t \rightarrow+\infty
$$

Follows from Lemma 1, Lemma 2, Lemma 3 and Lemma 4, $\Phi$ related to (16) possesses a $\mathcal{D}$ pull-back random attractor $\mathcal{A}(\tau, \omega) \subseteq \Lambda(\tau, \omega) \cap B_{0}(\omega)$. The proof is completed.

Acknowledgments: "The authors would like to thank the referee for improving the readability of the paper."

Author Contributions: All authors contributed equally to the writing of this paper. All authors read and approved the final manuscript.

Conflicts of Interest: "The authors declare no conflict of interest."

\section{References}

[1] Dafermos, C. M. (1970). Asymptotic stability in viscoelasticity. Archive for Rational Mechanics and Analysis, 37(4), 297-308.

[2] Borini, S., \& Pata, V. (1999). Uniform attractors for a strongly damped wave equation with linear memory. Asymptotic Analysis, 20(3-4), 263-277.

[3] Pata, V., \& Zucchi, A. (2001). Attractors for a damped hyperbolic equation with linear memory. Advances in Mathematical Sciences and Applications, 11, 505-529

[4] Ma, Q., \& Zhong, C. (2004). Existence of strong global attractors for hyperbolic equation with linear memory. Applied Mathematics and Computation, 157(3), 745-758.

[5] Park, J. Y., \& Kang, J. R. (2010). Global attractor for hyperbolic equation with nonlinear damping and linear memory. Science China Mathematics, 53(6), 1531-1539.

[6] Arnold, L., \& Chueshov, I. (1998). Order-preserving random dynamical systems: equilibria, attractors, applications. Dynamics and Stability of Systems, 13(3), 265-280.

[7] Flandoli, F., \& Schmalfuss, B. (1996). Random attractors for the 3D stochastic Navier-Stokes equation with multiplicative white noise. Stochastics: An International Journal of Probability and Stochastic Processes, 59(1-2), 21-45.

[8] Crauel, H., Debussche, A., \& Flandoli, F. (1997). Random attractors. Journal of Dynamics and Differential Equations, 9(2), 307-341.

[9] Temam, R. (1997). Infinite-dimensional dynamical systems in mechanics and physics. Springer-Verlag, New York.

[10] Wang, B. (2012). Sufficient and necessary criteria for existence of pullback attractors for non-compact random dynamical systems. Journal of Differential Equations, 253(5), 1544-1583.

[11] Fan, X. (2004). Random attractor for a damped sine-Gordon equation with white noise. Pacific Journal of Mathematics, 216(1), 63-76.

[12] Yin, F., \& Liu, L. (2014). D-pullback attractor for a non-autonomous wave equation with additive noise on unbounded domains. Computers \& Mathematics with Applications, 68(3), 424-438.

[13] Crauel, H., \& Flandoli, F. (1998). Hausdorff dimension of invariant sets for random dynamical systems. Journal of Dynamics and Differential Equations, 10(3), 449-474.

[14] Yang, M., Duan, J., \& Kloeden, P. (2011). Asymptotic behavior of solutions for random wave equations with nonlinear damping and white noise. Nonlinear Analysis: Real World Applications, 12(1), 464-478.

[15] Zhou, S., \& Zhao, M. (2015). Random attractors for damped non-autonomous wave equations with memory and white noise. Nonlinear Analysis: Theory, Methods \& Applications, 120, 202-226.

[16] Wang, Z., \& Zhou, S. (2015). Asymptotic behavior of stochastic strongly wave equation on unbounded domains. Journal of Applied Mathematics and Physics, 3(03), 338-357.

[17] Ball, J. M. (1973). Stability theory for an extensible beam. Journal of Differential Equations, 14(3), 399-418.

[18] Kang, J. R. (2011). Global attractor for an extensible beam equation with localized nonlinear damping and linear memory. Mathematical Methods in Applied Sciences, 34(12), 1430-1439.

[19] Park, J. Y., \& Kang, J. R. (2011). Uniform attractor for non-autonomous suspension bridge equations with localized damping. Mathematical Methods in Applied Sciences, 34(4), 487-496. 
[20] Xu, L., \& Ma, Q. (2015). Existence of random attractors for the floating beam equation with strong damping and white noise. Boundary Value Problems, 2015(1), 1-13.

[21] Pazy, A. (2012). Semigroups of linear operators and applications to partial differential equations (Vol. 44). Springer Science \& Business Media.

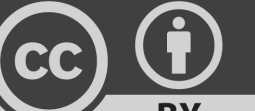

BY

(C) 2020 by the authors; licensee PSRP, Lahore, Pakistan. This article is an open access article distributed under the terms and conditions of the Creative Commons Attribution (CC-BY) license (http://creativecommons.org/licenses/by/4.0/). 